\title{
Leading Edge Noise Reduction of Thin Aerofoil by the Straight and Curved Serrations of the Add-on Type
}

\author{
Auris Juknevicius ${ }^{1}$, Tze Pei Chong ${ }^{2}$, Philip Woodhead ${ }^{3}$ \\ Brunel University London, Uxbridge, UB8 3PH, UK
}

\begin{abstract}
This paper presents the experimental results of the effect of add-on type leading edge serrations on the aeroacoustic performance of a symmetrical NACA0008 aerofoil. The focus is on the turbulence-leading edge interaction broadband noise. Tests have been conducted in an aeroacoustic open jet wind tunnel at Brunel University London. 25 serrated leading edges (straight) that can be adequately described by their serration wavelength $\lambda$ and serration amplitude $h$ have been investigated at Reynolds numbers between 0.2 and 0.6 millions. It was found that those with large $h$ are very effective in reducing the broadband noise levels up to about $9 \mathrm{~dB}$. However, the serration designs with smaller $h$ can produce noticeable levels of noise increase at high frequency. The effect of the $\lambda$ on the noise reduction depends on the corresponding $h$ value. For example, a particular design with both the largest $\lambda$ and $h$ has been shown to be very effective. Another one with a similar $\lambda$, but the smallest $h$ can actually degrade the performance significantly where noise increase over the baseline aerofoil becomes dominant. The curved-serration has been shown to be able to outperform the straight-serration counterpart by a further $5 \mathrm{~dB}$ broadband noise reduction when it is optimised properly. The mechanism is due to the increase of the "effective" serration amplitude $h^{\prime}$ as a result of the curvature although its "normal" serration amplitude $h$ actually remains the same as the straight-serration counterpart. Currently, there is no evidence to support the hypothesis that the peak of the curved-serration can prevent the interaction between the grid-generated turbulence structures and the serration troughs. Overall, the add-on type leading edge serration has been shown to be very effective in the reduction of the interaction broadband noise. The nature-inspired concept of a curvedserration at the leading edge provides an avenue for further research.
\end{abstract}

\section{Introduction}

$\mathbf{I}_{\mathrm{c}}^{\mathrm{t}}$ has been shown that noise generated by the industrial operations (e.g. aircraft and wind turbine) exerts considerable negative impact to the environment, and this has become an increasingly sensitive topic. In particular, the civil aeronautical industry is a substantial contributor to the noise pollution levels on a global scale and it is still receiving significant attention in terms of its impact on the communities surrounding the large airports. The UK Department for Transport forecasts that the current national passenger traffic of $\sim 254$ million is expected to exceed 335 million by 2030. Such forecasts therefore call for regulations to be implemented by the airports or local authorities as a result of related regional initiatives such as ACARE Flightpath 2050. This programme is backed by the European Commission, which sets goals to achieve 65\% reduction in commercial aviation noise emissions by

\footnotetext{
${ }^{1}$ PhD Student, Department of Mechanical, Aerospace and Civil Engineering, auris.juknevicius@ brunel.ac.uk, NonAIAA Member

${ }^{2}$ Senior Lecturer, Department of Mechanical, Aerospace and Civil Engineering, t.p.chong@ brunel.ac.uk, AIAA Member (corresponding author)

${ }^{3}$ PhD Student, Department of Mechanical, Aerospace and Civil Engineering, philip.woodhead@ brunel.ac.uk, AIAA Student Member
} 
2050 , relative to the level in year 2000. The aircraft and turbomachinary manufacturers are constantly improving the aerodynamic performance of their aircraft body. They are also developing the most advanced and efficient aeroengine that produces smaller carbon footprint and noise radiation level. To a large extent, the jet noise has been suppressed considerably due to the introduction of large bypass ratio aero-engine. As a result, other aircraft noise sources (e.g. airframe noise, fan noise and combustion noise) become increasingly important.

The wind turbine industry is another major player that actively seeks to develop the latest fan noise reduction technology for their turbine blades. This is especially important since large expansion of wind farms as a sustainable energy source in recent years has produced significant noise levels that the surrounding communities are exposed to. This, in turn, has been making the wind farm expansion an increasingly costly and complex process with the increasing need to construct such farms in less populated areas.

One of the important noise sources that are related to the above industries is the leading edge-turbulence interaction noise. It has been shown in the previous researches that leading edge noise is normally related to the convecting large turbulent structures in the freestream. These turbulence structures interact with the leading edge of an aerofoil before being stretched around it, and in the process induce large pressure fluctuations on the suction and pressure sides of the aerofoil. Significant level of broadband noise radiation is produced as a result of the amplified unsteady lift ${ }^{1}$. To reduce the leading edge noise, one of the most effective methods is to apply the owl-inspired serrated pattern at the leading edge. Some researches carried out on owl's silent flight (mainly measurements of mid-flight noise emissions) have led to postulations that these serrations could be partially responsible for the unique in-flight noise reduction capability of an owl. The following literature review will briefly summarise the use of the serration technology for the aerofoil noise reduction.

In the 1970,'s, the effect of the leading edge serration originally tested by Soderman et al. ${ }^{2}$ was further investigated by Hersh and Hayden ${ }^{3}$ for its ability to reduce aerofoil tonal noise from helicopter blades. They showed that leading edge serrations can achieve noise reduction between 4-8 dB. Since then, there is renewed interest recently to apply leading edge serration to achieve the aerofoil turbulence-leading edge interaction noise reduction. In a joint experimental-numerical efforts by Clair et al. ${ }^{4}$ on the NACA 65-(12)10 aerofoil, the serrated leading edge is found to achieve broadband noise reduction between 3-4 dB. Narayanan et al. ${ }^{5}$ assess the effects of a serrated leading edge on the turbulence-leading edge interaction noise for a flat plate, as well as a NACA 65-(12)10 aerofoil. A near isotropic turbulence was produced using mesh grid inside the nozzle. They demonstrated significant broadband noise reduction of $9 \mathrm{~dB}$ by flat plate and $7 \mathrm{~dB}$ by aerofoil configurations, respectively, using the largest serration amplitude. They suggested that the level of broadband noise reduction is a strong function of the serration amplitude $h$, and is less sensitive to the serration wavelength $\lambda$. An inviscid numerical study by Kim et al. ${ }^{6}$ exhibited a de-correlation of the surface pressure fluctuation and the far field noise on a serrated leading edge. In particular, the noise source at the mid-region of the oblique edge becomes ineffective across the mid to high frequency range. Another noise reduction mechanism is attributed to the phase interference and destruction effect between the serration peak and the mid-region of the oblique edge. Apart from the $h$ and $\lambda$, the serrated leading edge is also found to be sensitive to other influencing parameters such as the Reynolds number, turbulence intensity and angle of attack. These interdependencies of factors were studied empirically by Biedermann et al. ${ }^{7}$ to model the serrated leading edge noise.

So far, the leading edge device for the reduction of interaction broadband noise is almost exclusively formed by the cut-in approach, i.e. the serration pattern is cut into the main body of the aerofoil. The design and manufacturing complexities of the cut-in serration is clearly the disadvantages. The next technological advance for the leading edge serration is to simplify the design and manufacturing processes, thus making it easier to be implemented in an aerofoil. In contrast to the cut-in approach, the leading edge serration can also be the add-on type (just like its trailing edge serration counterpart). The first objective of this paper is to address whether an add-on serrated leading edge, which has clear advantages owing to their relative simplicity and ability to apply to most of the thin aerofoil, can produce the similar level of interaction broadband noise reduction as the cut-in type.

As observed by the ornithologists, the leading edge of an owl's wing exhibits a comb-like "curved" serration. The curvature adds at least two additional geometrical parameters beside the $h$ and $\lambda$. In particular, the curved-flow path within the sawtooth gap will produce an effective serration amplitude $h^{\prime}$, which is normally longer than the $h$ counterpart for an otherwise straight serration. The second objective of this paper, therefore, is to exploit this unique morphology of the owl wing for the leading edge serration on an aerofoil. As pointed out by Kim et al. ${ }^{6}$, the remaining contributor to the interaction noise radiation for a serrated leading edge is related to the flow dynamics at the sawtooth trough region. Because each consecutive sawtooth tips of a curved leading edge serration exhibits a spanwise offset to the sawtooth trough at the downstream, the incoming turbulence structures could be shielded by the sawtooth tip. This has a potential to reduce the turbulence level at the trough, which then leads to a further reduction of the broadband interaction noise level. 

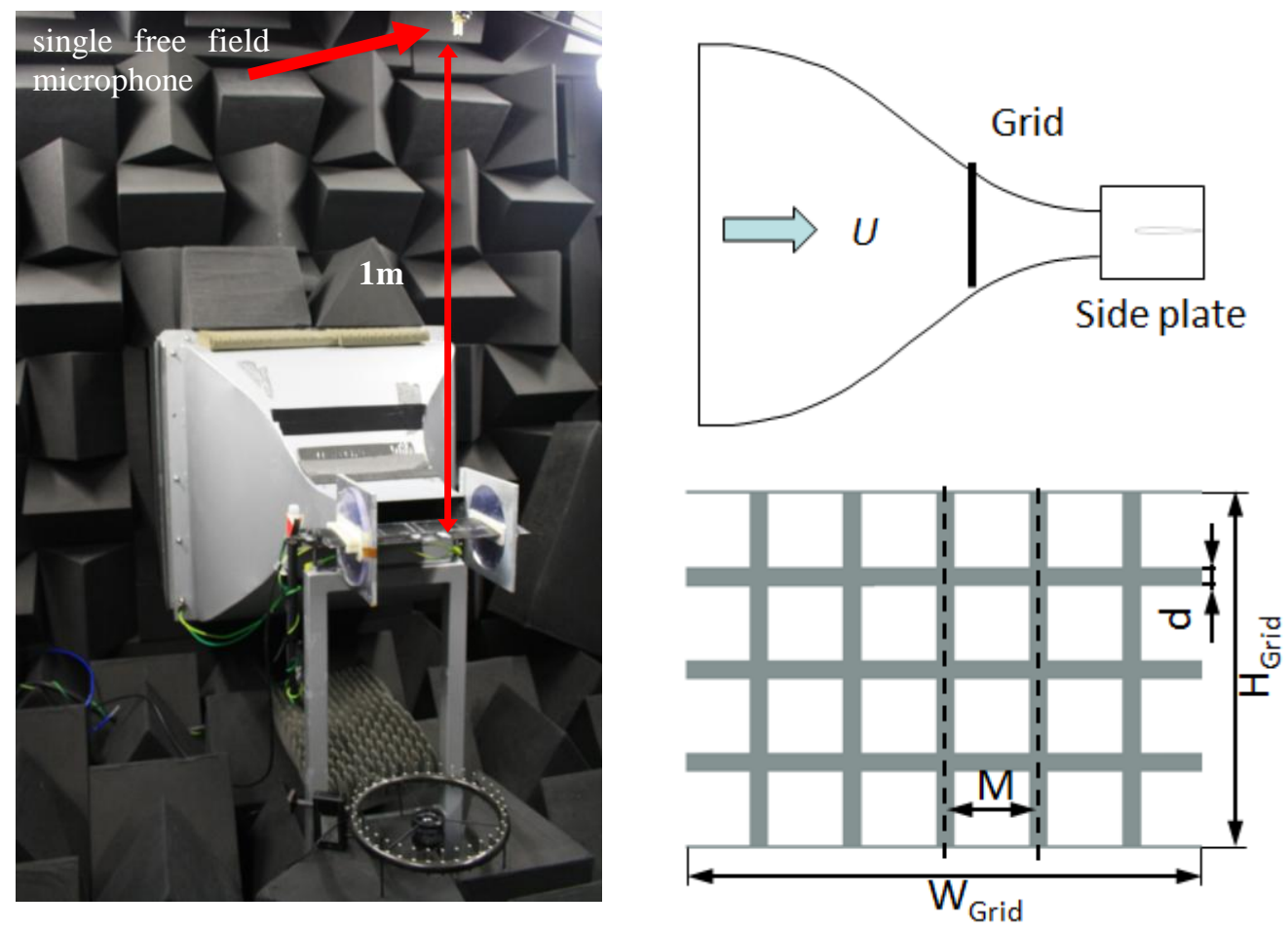

Fig. 1 A typical set up for the aerofoil noise experiments at the Brunel aeroacoustic wind tunnel for the turbulence-leading edge interaction noise. The placement of the turbulence bi-planar grid with relative to the nozzle is shown.

\section{Experimental setup}

\section{A. Wind tunnel facilities, instrumentations and the grid-generated turbulence}

Free field measurements of the aerofoil noise were conducted in the aeroacoustic open jet wind tunnel at Brunel University London, which is situated in a $4 \mathrm{~m} \times 5 \mathrm{~m} \times 3.4 \mathrm{~m}$ anechoic chamber. As shown in Fig. 1, the nozzle exit is rectangular with dimensions of $0.10 \mathrm{~m}$ (height) $\times 0.30 \mathrm{~m}$ (width). This wind tunnel can achieve a turbulence intensity of between $0.1-0.2 \%$ and a maximum jet velocity of about $80 \mathrm{~ms}^{-1}$. The background noise of the wind tunnel facility is well below the self noise of the quietest aerofoil across the whole range of velocity ${ }^{8}$. The range of jet speeds under investigation was $20 \leq U_{\infty} \leq 60 \mathrm{~ms}^{-1}$, which correspond to the chord-based Reynolds numbers $2 \times$ $10^{5} \leq \operatorname{Re} \leq 6 \times 10^{5}$, respectively. The aerofoil chord length is $0.15 \mathrm{~m}$. The aerofoil was held by side plates and attached flushed to the nozzle lips. Unless otherwise stated, the aerofoil was always positioned at an angle of attack $\alpha=0^{\circ}$ with relative to the main jet direction.

Also shown in Fig. 1 is far field noise measurements were made by a single condenser microphone at polar angles of about $90^{\circ}$ at a distance of $1.0 \mathrm{~m}$ from the aerofoil leading edge at mid span. Noise data was acquired at a sampling frequency of $44 \mathrm{kHz}$ for 15 seconds by a 16-bit Analogue-Digital card from National Instrument. The data was then windowed and the Power Spectral Density (PSD) of $1 \mathrm{~Hz}$ bandwidth was computed from a 1024 point Fast Fourier Transform.

To generate large scale turbulence structures, and subsequently the elevated level of turbulence intensity in the freestream, a bi-planar orthogonal square grid was placed inside the nozzle as shown in Fig. 1. The mesh length is $75 \mathrm{~mm}$ and the grid diameter is $15 \mathrm{~mm}$. Hot-wire measurement of the freestream turbulence intensity and eddy length scale at location near the location of the aerofoil leading edge (but without the presence of the aerofoil) are $3.7 \%$ and $6.5 \mathrm{~mm}$, respectively. In our previous publications ${ }^{7,9}$, the above bi-planar grid has been demonstrated to achieve a reasonably isotropic turbulence as the normalised turbulence energy spectra of the fluctuation velocity 
measured by the hot-wire agreed well with the Von Karman and Liepmann's one-dimensional turbulence model. The comparison is not shown here.

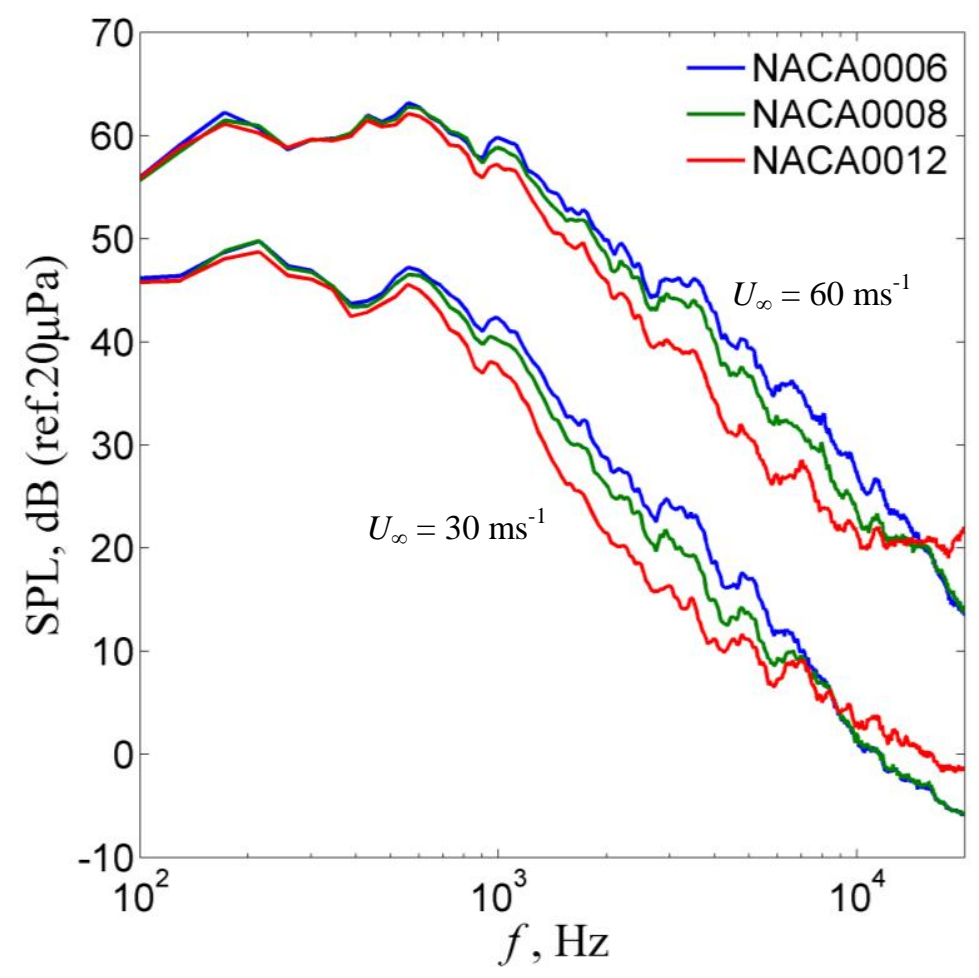

Fig. 2 Sensitivity of the SPL spectra produced by NACA00series aerofoil of different thicknesses.

\section{B. Selection of the aerofoil type}

The serrated leading edge inserts used in this study have a thickness of about $0.8 \mathrm{~mm}$. In order to maintain the ratio of thicknesses between the serration inserts and the aerofoil leading edge to be at least 0.5 , a thin aerofoil is more suitable. A thin aerofoil also features a large curvature at the leading edge. This means that, in comparison to a thicker aerofoil, the incoming turbulence structures to a thin aerofoil will be deprived of the mechanism of acceleration around the leading edge. As a result, high level of broadband noise will be radiated due to the significant turbulence-solid structure interaction. This particular point is demonstrated in Fig. 2 for the comparison of the noise spectra between the NACA0006, NACA0008 and NACA0012 symmetric aerofoils at $U_{\infty}=30 \mathrm{~ms}^{-1}$ and $60 \mathrm{~ms}^{-1}$, all of which were positioned at $\alpha=0^{\circ}$. The spectra in the figure are dominated by the turbulence-leading edge interaction characteristics, using the same bi-planar turbulence grid as discussed previously in Section A.

The figure demonstrates that the level of broadband noise increases with the reduced aerofoil thickness (NACA0006 has the maximum thickness of $6 \%$ of the chord, the NACA0008 $=8 \%$ and the NACA0012 $=12 \%$ ). Thin aerofoil is commonly adopted in the OGV (Outlet Guided Vanes) configuration, or in a compressor cascade. Since very few researches focus on the investigation of noise on thin aerofoils, the NACA0008 aerofoil was selected in this study. As shown in Fig. 3, the maximum aerofoil thickness is $12 \mathrm{~mm}$ (8\% of $0.15 \mathrm{~m}$ for the chord length) and the span of the aerofoil is $b=0.496 \mathrm{~m}$. Note that during the noise measurement, only $b=0.3 \mathrm{~m}$ of the aerofoil was submerged in the jet flow, where the excess parts were extended beyond the side plates of the open jet nozzle. The full width of the aerofoil will also be utilised during the measurement of the aerodynamic forces at a closed-working section wind tunnel. The results of the aerodynamic forces subjected to the add-on serrated leading edge are not included in this paper, but they will be reported elsewhere.

The aerofoil has been designed so that the first $25 \mathrm{~mm}$ of the body (starting from the leading edge) can be detached. Thus, one of the detachable leading edges provides a straight leading edge (i.e. without slot at the nose) to serve as a "clean" aerofoil shape, hereafter referred to as the baseline aerofoil ( $\lambda 0 h 0$ - the basis of the naming will 


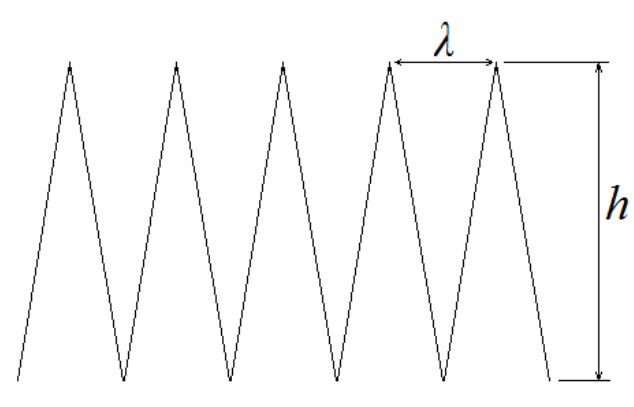

(a)

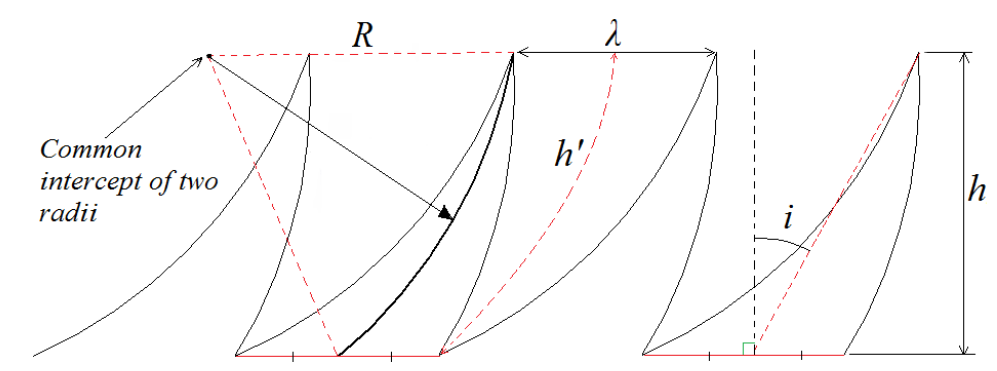

(b)

Fig. 3 Geometrical parameters for a (a) straight-serration, and (b) curved-serration

be discussed in Section C). The other detachable leading edge incorporates a slot with thickness $=0.8 \mathrm{~mm}$ and inner-length of $17 \mathrm{~mm}$ that runs along the chord line of the aerofoil. The slot allows various serration inserts to be slotted in and interchanged with other inserts easily. The flat plate serration inserts were laser-cut precisely. Cardboard sheets of about $0.8 \mathrm{~mm}$ thickness have been used. This material can achieve sufficient accuracy in terms of the serration dimensions, thin but also provides the required stiffness to avoid fluttering during experiments.

\section{Definition of the leading edge serrations}

The first type of serrations is the regular, straight sawtooth serrations shown in Fig. 3a. To gain a better understanding of the effect of serration geometry on the aeroacoustic performance, a wide range of serration geometries was designed. There are two main design parameters: serration wavelength $\lambda$ and serration amplitude $h$ as shown in Fig. 3a. The serration dimensions that have been chosen are $(5 \times \lambda)=2.5,5,10,15$ and $20 \mathrm{~mm}$, and $(5 \times$ $h)=5,10,15,20$ and $30 \mathrm{~mm}$. The total combination is therefore 25 serrated leading edges (straight). Note that the naming of each serrated leading edge, including the baseline case, is in accordance to the values of their serration wavelength $\lambda$, and serration amplitude $h$. For example, the "clean", baseline leading edge mentioned in Section B, which does not have contain any serration wavelength and serration amplitude, is therefore named as $\lambda 0 h 0$. Likewise, the serrated leading edge that has a serration wavelength of $2.5 \mathrm{~mm}$ and serration amplitude of $30 \mathrm{~mm}$ will be named as $22.5 h 30$. In one instance (beginning of Section III), noise results produced by flat plate add-on of straight, unserrated profile are also presented. This type of baseline configuration with flat plate add-on will be termed as $\lambda 0 h \mathrm{XX}$, where $\mathrm{X}$ is the longitudinal length of the flat plate add-on.

For the curved-serrations, they were manufactured in a similar way as the straight serration. As shown in Fig. 3b, the curved serration designs are based on five main geometric parameters: the serration wavelength $\lambda$, serration amplitude (straight) $h$, serration amplitude (curved) $h^{\prime}$, inclination angle $i$ and curvature radius $R$ (where larger serration curvature is characterised by a smaller radius $R$ ). In the current study, the curved-serrations are designed to have two inclination angles $i=15^{\circ}$ and $30^{\circ}$, and two radii $R=50 \mathrm{~mm}$ and $100 \mathrm{~mm}$. These provide a total of four types of curved serration for each combination of $\lambda$ and $h$. These variations in $i$ and $R$ allow for a reasonable wide range of geometrical change, thus allowing the generalisation of the curvature effect on the aeroacoustic performances. It is also worth pointing out that the values for $i=15^{\circ}$ and $R=50 \mathrm{~mm}$ provide a serration pattern that is quite similar to those found in the owl's wings. The basis of the naming for the curved-serration is also quite similar. For example, for a combination of $\lambda=2.5 \mathrm{~mm}$ and $h=30 \mathrm{~mm}$, and with $i=15^{\circ}$ and $R=50 \mathrm{~mm}$, it will be named as $\lambda 2.5 h 30\left(i 15^{\circ} R 50\right)$. A straight serration of the same combination of $\lambda$ and $h$ will either be named as $\lambda 2.5 h 30$ (straight), or simply be $\lambda 2.5 h 30$.

\section{General Aeroacoustic Characteristics for the Straight-Serrated Leading Edges}

This section will present the experimental results pertaining to the broadband noise reduction by the straightserrated leading edges. Before that, it is important to define the correct baseline configuration where all the serrated leading edges will be compared against. There are two possible choices. The first choice is the $\lambda 0 h 0$ type where no flat plate insert is present at the leading edge. The second choice is the $\lambda 0 h \mathrm{XX}$ type where a flat plate insert of 


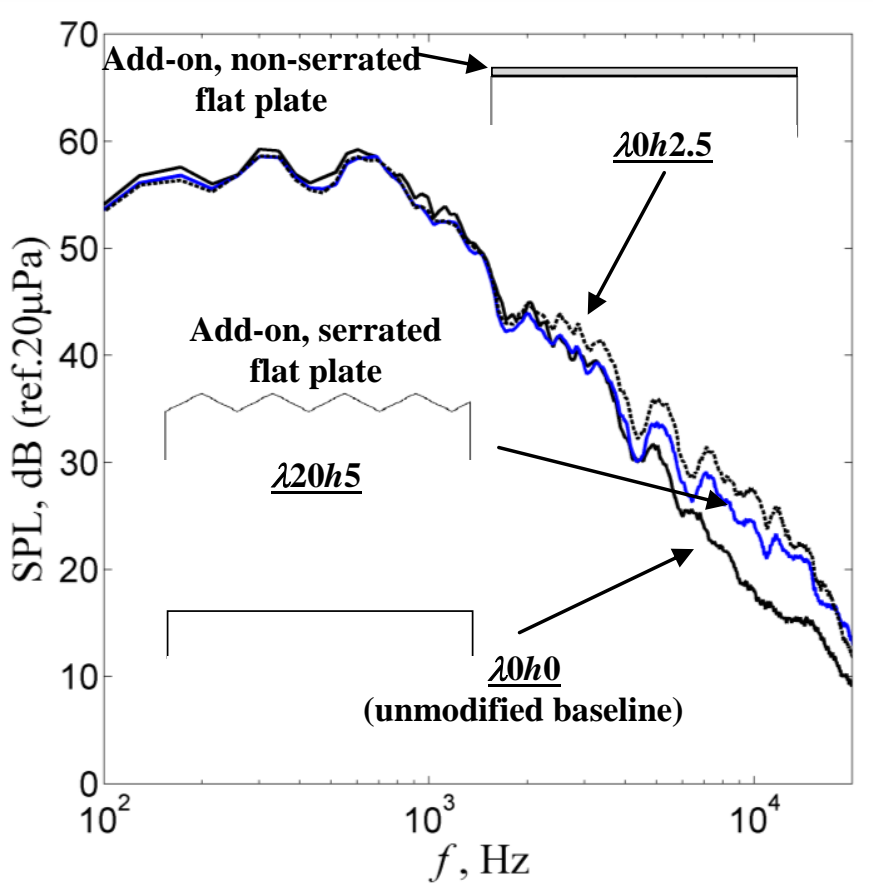

(a)

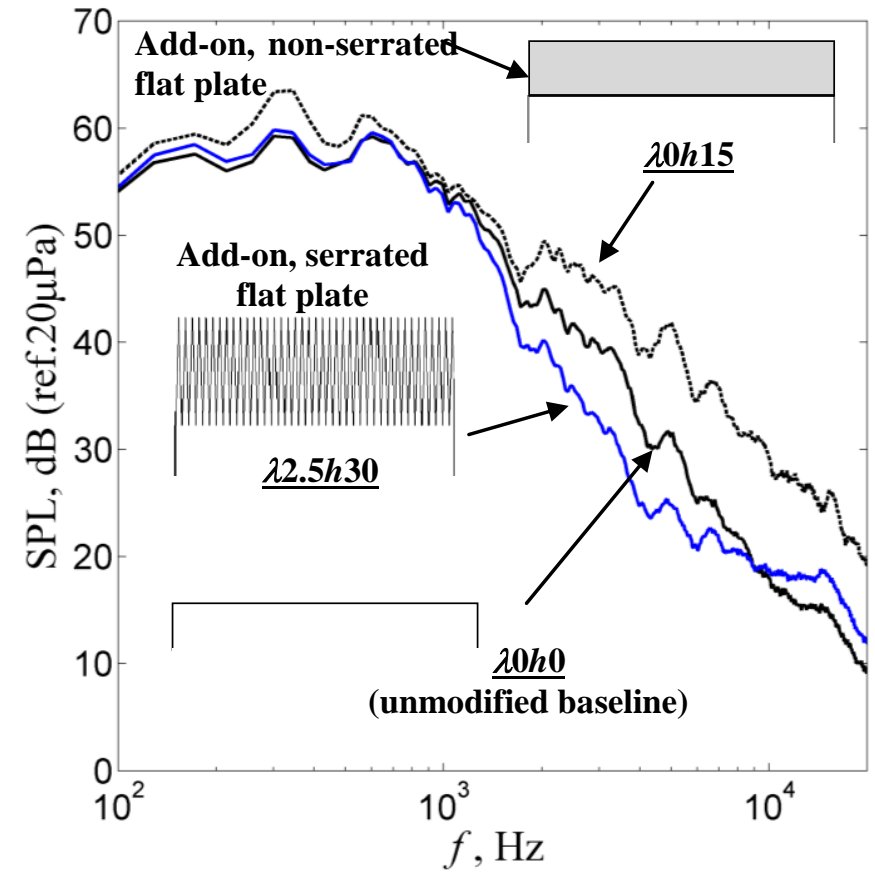

(b)

Fig. 4 Comparison of the SPL spectra produced by the unmodified baseline $(\lambda 0 h 0)$, and the (a) baseline with add-on flat plate $(\lambda 0 h 2.5)$ and add-on serrated leading edges ( $\lambda 20 h 5)$, and (b) baseline with add-on flat plate $(\lambda 0 h 15)$ and add-on serrated leading edges $(\lambda 2.5 h 30) . U_{\infty}=50 \mathrm{~ms}^{-1}$.

unserrated, straight profile is attached to the leading edge. The second choice has a feature where its longitudinal length will be half of that of the corresponding add-on serrated leading edge in order to match the same wetted area. For example, a serrated leading edge with $h=30 \mathrm{~mm}$ will be compared against a straight, add-on baseline leading edge of $h=15 \mathrm{~mm}$ (e.g. $\lambda 2.5 h 30$ vs. $\lambda 0 h 15)$, and so on.

Figure 4a shows the Sound Pressure Level (SPL) spectra for the $\lambda 0 h 0, \lambda 20 h 5$ and $\lambda 0 h 2.5$ cases at $U_{\infty}=50 \mathrm{~ms}^{-1}$ (corresponds to $\mathrm{Re}=5 \times 10^{5}$ ). From the figure, the SPL produced by the serrated $\lambda 20 h 5$ does not vary significantly with the $\lambda 0 h 0$ baseline case at $<4 \mathrm{kHz}$. Above this frequency, however, the $\lambda 20 h 5$ is consistently higher in the SPL than the $\lambda 0 h 0$. This means that when the serrated $\lambda 20 h 5$ is compared against the $\lambda 0 h 0$, not only it is inefficient to reduce the broadband noise, but it can also be up to $8 \mathrm{~dB}$ noisier at high frequency. However, a complete different story entails when the $\lambda 20 h 5$ is compared against the flat plate baseline $\lambda 0 h 2.5$. For this comparison, the serrated $\lambda 20 h 5$ can achieve broadband noise reduction across a large range of frequency ( $>2 \mathrm{kHz}$ ). When another add-on serrated leading edge is used, in this case the $\lambda 2.5 h 30$, a similar trend can also be observed in Fig. $4 \mathrm{~b}$. The $\lambda 2.5 h 30$ serrated leading edge, which will later be confirmed as one of the most efficient in terms of broadband noise reduction, is shown to outperform the $\lambda 0 h 0$ clean baseline, but even more significantly for the $\lambda 0 h 15$ add-on baseline case.

By adding an unserrated, straight flat plate insert to the aerofoil leading edge will increase the noise level. The noise level is also found to increase when the longitudinal length of the flat plate is increased. It is clear that this cannot be the meaningful baseline case to be compared against other add-on serrated leading edges because the level of broadband noise reduction will be inflated artificially. After all, the industrial fan blades are unlikely to install the unserrated flat plate on their leading edges in the first place. Therefore, the true baseline case should be the $\lambda 0 h 0$, and this forms the basis of noise comparison throughout the paper.

The results in Fig. 4 also provided a hint of the sensitivities of the $\lambda$ and $h$ to the level of broadband noise reduction. Here the level of noise reduction is denoted as $\Delta \mathrm{SPL}$, which is defined as $\operatorname{SPL}(\lambda 0 h 0)-\operatorname{SPL}(\lambda x h y)$, where $x \in 2.5,5,10,15$ or $20 \mathrm{~mm}$, and $y \in 5,10,15,20$ or $30 \mathrm{~mm}$. A positive value of $\Delta S P L$ represents reduction in noise by the serrated leading edge, and the opposite is true. Large amount of data are gathered for the $\Delta$ SPL spectra 


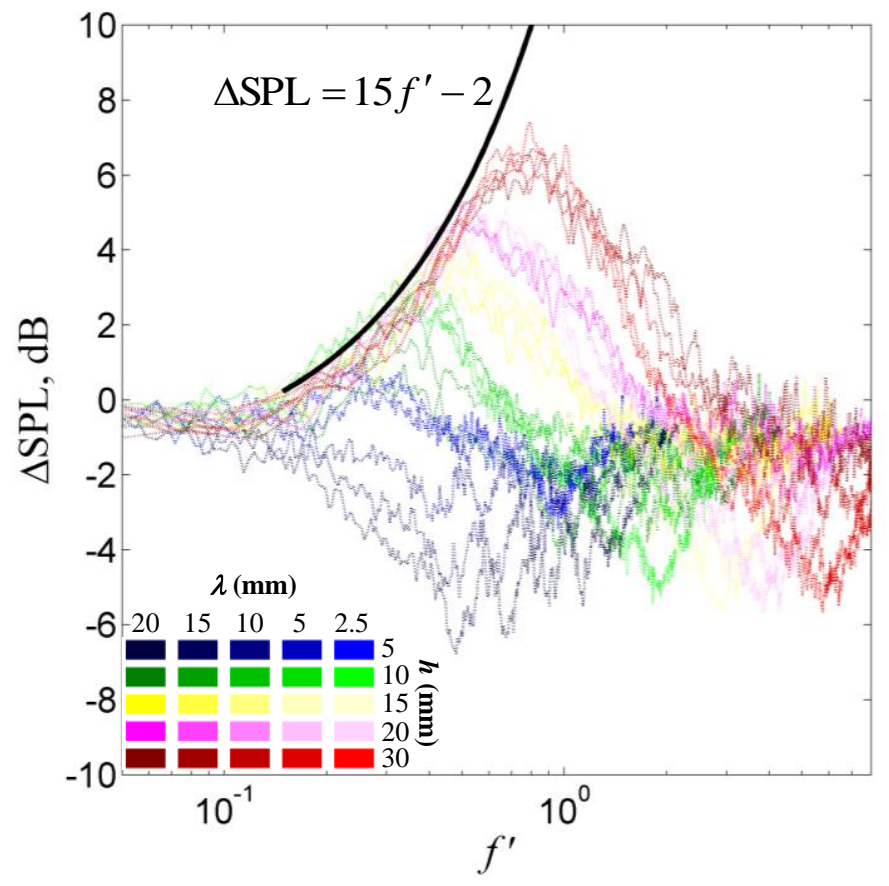

(a)

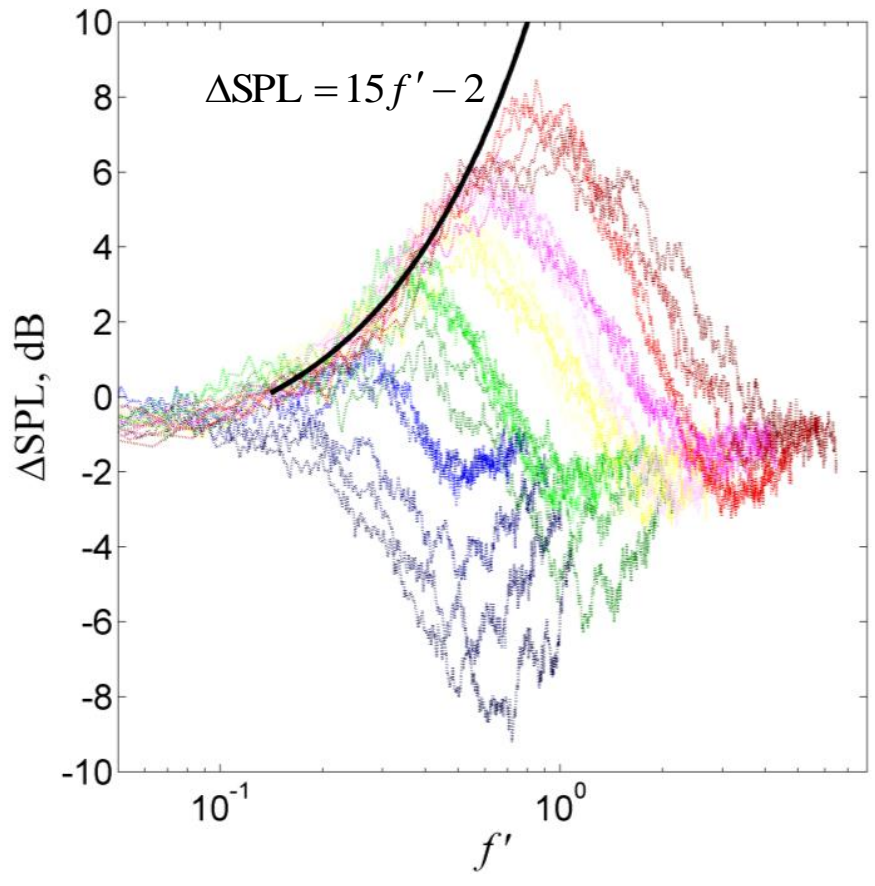

(b)

Fig. $5 \Delta \mathrm{SPL}$ (dB) spectra produced by the serrated leading edges of different $\lambda$ and $h$ at (a) $\operatorname{Re}=$ $3 \times 10^{5}\left(U_{\infty}=30 \mathrm{~ms}^{-1}\right)$, and $(b) \operatorname{Re}=6 \times 10^{5}\left(U_{\infty}=60 \mathrm{~ms}^{-1}\right)$. Note that $f^{\prime}=f h / U_{\infty} .1 / \log (\lambda)$.

following the experiments, which cover the $25 \times$ serrated leading edges at $2 \times 10^{5} \leq \operatorname{Re} \leq 6 \times 10^{5}$. After analysing all the data set, the $\triangle \mathrm{SPL}$ for the leading edge broadband noise is found to follow a simple linear function as follow:

$$
\Delta \mathrm{SPL}=-15 f^{\prime}-2, \quad \text { where } f^{\prime}=\frac{f h}{U_{\infty}} \cdot \frac{1}{\log (\lambda)} \text { and } \lambda<1 \mathrm{~m}
$$

Some examples are illustrated in Fig. 5a and Fig. $5 \mathrm{~b}$ for the $\operatorname{Re}=3 \times 10^{5}$ and $6 \times 10^{5}$, respectively. The use of $f^{\prime}$ is found to provide a collapse of the curves amongst the serrated leading edges for the $f^{\prime}$ range just before it reaches the maximum level of $\Delta$ SPL. The subsequent roll-off of the $\Delta$ SPL following the $f^{\prime}{ }_{\text {(peak) }}$ simply means that the leading edge noise is becoming less dominant at higher frequency, and as a result, the serrated leading edge devices also become less effective in reducing the noise. Based on the literatures for the cut-in type serrated leading edges ${ }^{5,7}$, ${ }^{9}$, the serrated leading edge is the most effective in achieving the $\Delta$ SPL when the $\lambda$ is small and the $h$ is large. The use of $1 / \log (\lambda)$ in EQ. (1), with $\lambda<1 \mathrm{~m}$, seems counter-intuitive as this entails that a larger $\Delta$ SPL is achieved with a larger $\lambda$. However, previous attempts of different scaling parameters $\frac{f h}{U_{\infty}} \cdot \log (\lambda)$, or simply the $\frac{f h}{U_{\infty}}$ failed to collapse the curves for all the serrated leading edge devices.

It is interesting to examine the behaviour of the $\triangle$ SPL across a wide range of Re achieved by the 25 serrated leading edges with different combinations of $\lambda$ and $h$. This is shown in Fig. 6 as the $\Delta$ SPL contour maps. It is apparent that the two governing geometrical parameters of the serrations, $\lambda$ and $h$, can yield different noise reduction capabilities for the serrated aerofoil. It was found that the highest $\Delta$ SPL is usually associated with small $\lambda$ and large $h$.

Two extreme serration cases are compared here: the $\lambda 2.5 h 30$ versus $\lambda 20 h 5$. Serration $\lambda 2.5 h 30$ achieved the largest $\Delta$ SPL in the range of $7-9 \mathrm{~dB}$. Between $2 \times 10^{5} \leq \mathrm{Re} \leq 6 \times 10^{5}$, the frequency range covering the $+\Delta \mathrm{SPL}$ is 0.3 $\mathrm{m}^{-1} \leq f^{\prime} \leq 1.9 \mathrm{~m}^{-1}$. The mechanism through which the broadband noise reductions are achieved for the $\lambda 2.5 h 30$ is likely to be associated with the serrations acting as the 'filter' and breakup device for the incoming turbulent flow structure. This point is illustrated in the hypothetical schematic of Fig. 7, which shows that as the incoming turbulent 

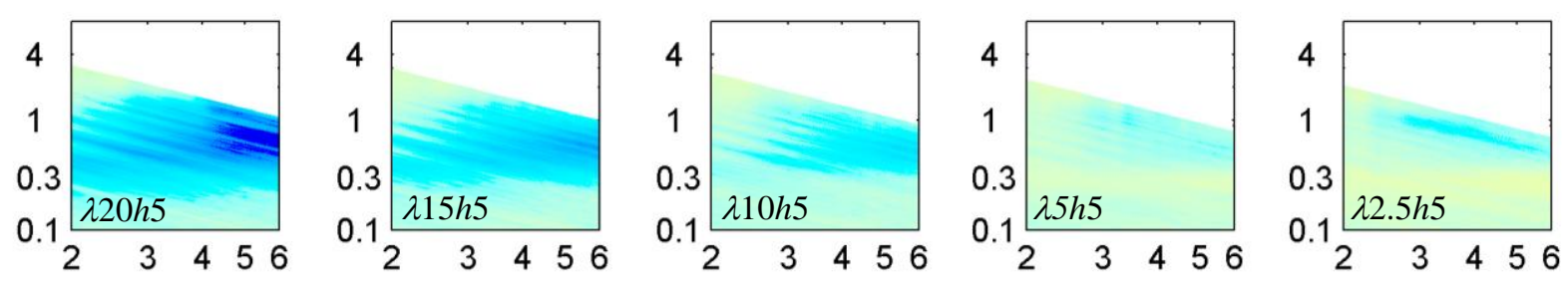

$\triangle \mathrm{SPL}$
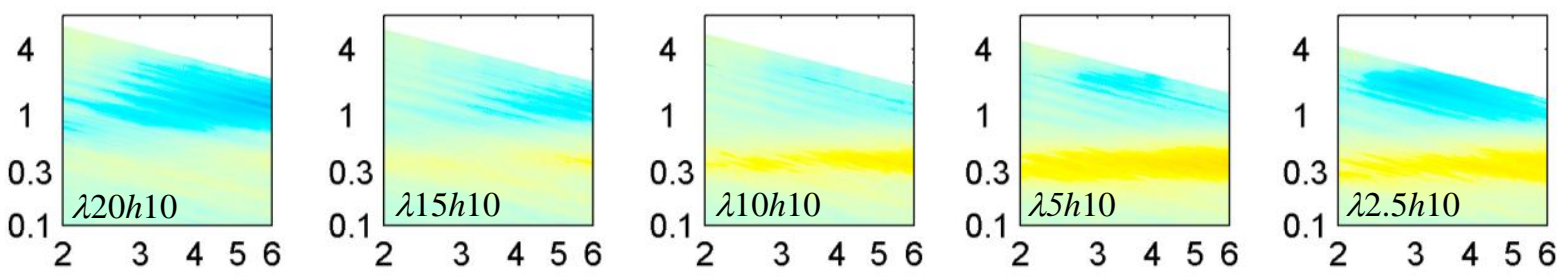
$(\mathrm{dB})$
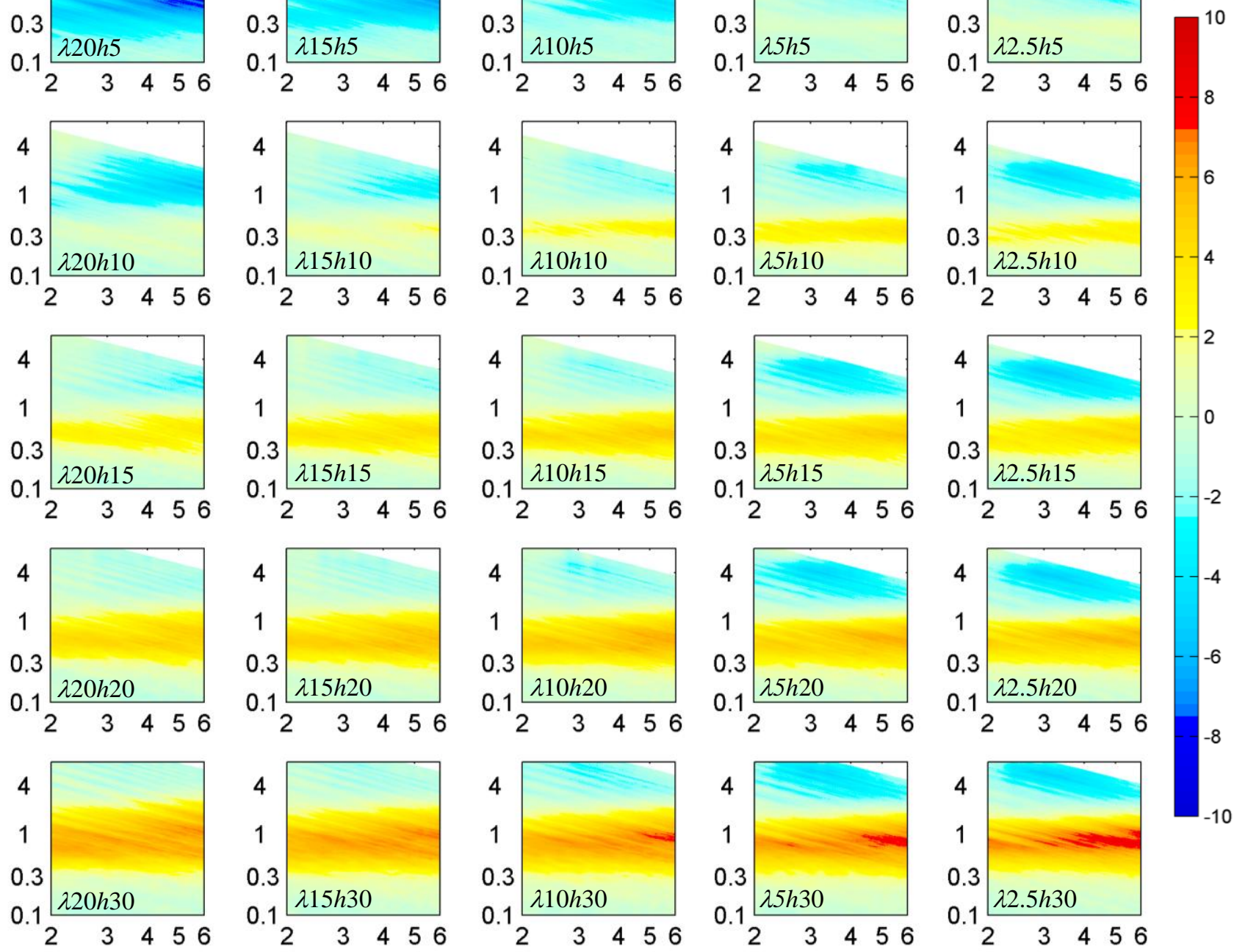

Fig. 6 Contour maps of the $\Delta$ SPL (dB) produced by different combinations of $\lambda$ and $h$ for the serrated leading edges. The abscissa is the $\operatorname{Re}\left(\times 10^{5}\right)$, and the ordinate is the $f^{\prime}$.

flow structures impinge the sawtooth of the $\lambda 2.5 h 30$, smaller eddies of length scale equivalent to the $\lambda$ are brokenup. With a small $\lambda$, the sharp serration angle will force the turbulent eddies to 'stretch' and break-up further in the direction of the main flow. Therefore by the time the smaller-scale turbulent eddies reach the troughs, the lower level of turbulence intensity will become less effective in the broadband noise radiation.

The other extreme serration case is the $\lambda 20 h 5$ serrated leading edge, where significant noise increase between -5 and $-10 \mathrm{~dB}$ at $f^{\prime}>0.3 \mathrm{~m}^{-1}$ has been demonstrated. The level of noise increase is found to be more significant at high Re. For example, the $-\Delta \mathrm{SPL}$ at $\mathrm{Re}>4.5 \times 10^{5}$ is between -9 and $-10 \mathrm{~dB}$. Again, the mechanism causing the high noise increase for the $\lambda 20 h 5$ case might be related to the specific responses of the turbulence structures to the serration geometry. By referring to the schematics in Fig. 7, an assumption is made that the same turbulence structures as the $\lambda 2.5 h 30$ case are also present and are convecting downstream of the $\lambda 20 h 5$ case. Between $5 \mathrm{~mm}$ and $30 \mathrm{~mm}$ upstream of the trough the intensity of the grid-generated turbulence structures decay with the streamwise distance $^{10}$ at a rate of approximately power of -5 . This is the only dissipation mechanism of the turbulence within this region for the $\lambda 20 h 5$ case. Furthermore, neither the mechanisms of forced breakup, nor the stretching of the turbulence eddies is available to further reduce the intensity of the turbulence. However, the increased wetted length 


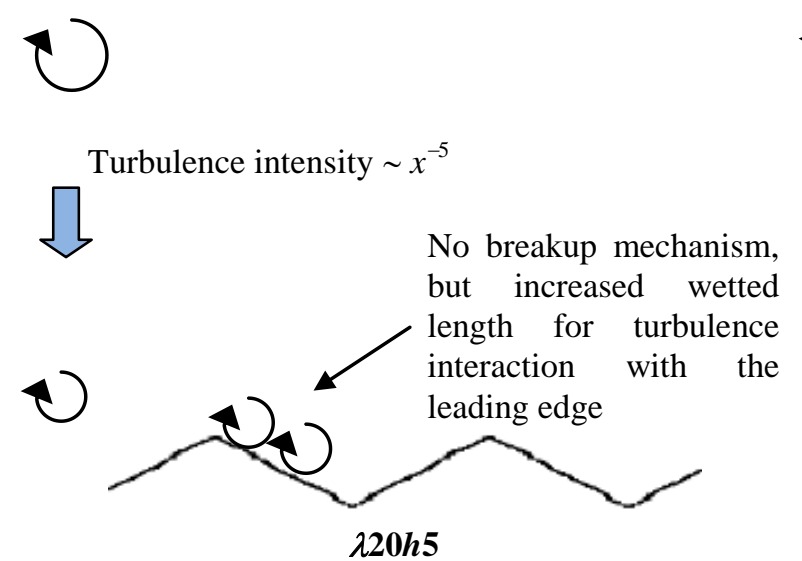

Fig. 7 Schematic hypothesising the change of the turbulence structure with relative to the different serration sawtooth geometries and their implication to noise reduction / increase

of the serration for the $\lambda 20 h 5$ case (as a result of the large serration angle and short serration amplitude) rather provides an enhanced interaction with the turbulence structures compared to the baseline $20 h 0$. As a result, the $\Delta \mathrm{SPL}$ becomes predominantly negative.

In Fig. 6, another serration case $220 h 30$ demonstrates outstanding noise performance as it has been shown to provide $\Delta$ SPL of 4-7 dB in a broader frequency range (compared to the $\lambda 2.5 h 30$ case) of $0.3 \mathrm{~m}^{-1} \leq f^{\prime} \leq 3.0 \mathrm{~m}^{-1}$ between $\operatorname{Re}=2 \times 10^{5}$ and $6 \times 10^{5}$. Furthermore, unlike the $\lambda 2.5 h 30$, the $\lambda 20 h 30$ serration design does not produce noticeable noise increase at the high frequency, which seems like a common feature for the serrated leading edge with small value of $\lambda$. This might be caused by the enhanced interaction between the turbulence eddies and the sawtooth oblique edges. As the $h$ of the serrations is increased, the relative spanwise distance between the successive serrations is decreased. Therefore, the incoming turbulent flow is forced to pass through many narrow passages, and generating high frequency interaction noise in the process. In contrary, for the $\lambda=20 \mathrm{~mm}$ case but with the same $h$, due to the larger relative spanwise distance between the successive serrations, the total number of the air passage is reduced and the turbulence interaction noise with the oblique side edges of the sawtooth is less significant.

It is generally observed that the level of noise reduction is the most sensitive to the serration amplitude $h$. To examine the overall effect, the overall sound pressure level OASPL is calculated for all the serrated leading edge cases, including the baseline $20 h 0$. The OASPL is obtained by integrating the mean-square acoustic pressure from $50 \mathrm{~Hz}$ to $20,000 \mathrm{~Hz}$. Here the level of OASPL reduction is represented by $\triangle$ OASPL, which is defined as $\operatorname{OASPL}(\lambda 0 h 0)-\operatorname{OASPL}(\lambda x h y)$, where $x$ and $y \neq 0$. Figure 8 shows the various $\Delta$ OASPL achieved by all the serrated leading edges. Similarly, a positive $\triangle$ OASPL denotes noise reduction, and the opposite is true.

It can be seen that the sensitivity of the noise performance with relative to the $h$ gradually decreases when the $\lambda$ is reduced. The $\triangle$ OASPL plots provide a good indication on the overall performance of the serrations. It can be seen that although the serration $22.5 h 30$ has shown the largest level of noise reduction in terms of SPL, it can only achieve the maximum $\triangle$ OASPL of about $1.5 \mathrm{~dB}$ at $\mathrm{Re}=2 \times 10^{5}$. This is because the OASPL includes the contribution of the extraneous noise at high frequency. As a result, the overall noise performance of this particular serration design is reduced. Another serration $\lambda 20 h 30$, however, can achieve an improved $\triangle$ OASPL of about $3.4 \mathrm{~dB}$ at the same Re. This is due to the fact that high frequency noise increase is absence for this particular serration design. The $\triangle$ OASPL results also highlight another observation in terms of the noise reduction capability. It can be seen that the serrations tend to undergo decrease in performance with increasing $U_{\infty}$ or Re. For the best performing serration $\lambda 20 h 30$, the $\triangle$ OASPL decreases from $3.4 \mathrm{~dB}$ at $\operatorname{Re}=2 \times 10^{5}$ down to $0.9 \mathrm{~dB}$ at $\operatorname{Re}=6 \times 10^{5}$. For the serrations with the same $h$, the sensitivity of the $\triangle$ OASPL with Re becomes larger for the large $\lambda$.

It has been shown that the most optimised leading edge serration that can achieve very large reduction in the broadband noise level, and yet does not produce noise increase at high frequency, is the $\lambda 20 h 30$ type. This particular serration is therefore chosen for the investigation of the effect when the aerofoil is subjected to different geometrical angles of attack $\alpha$. Note that the geometrical angle of attack $\alpha$ does not represent the true angle of attack due to the open jet deflection. Noise measurements were taken with the aerofoil placed in the freestream flow at $0^{\circ} \leq \alpha \leq 10^{\circ}$ 

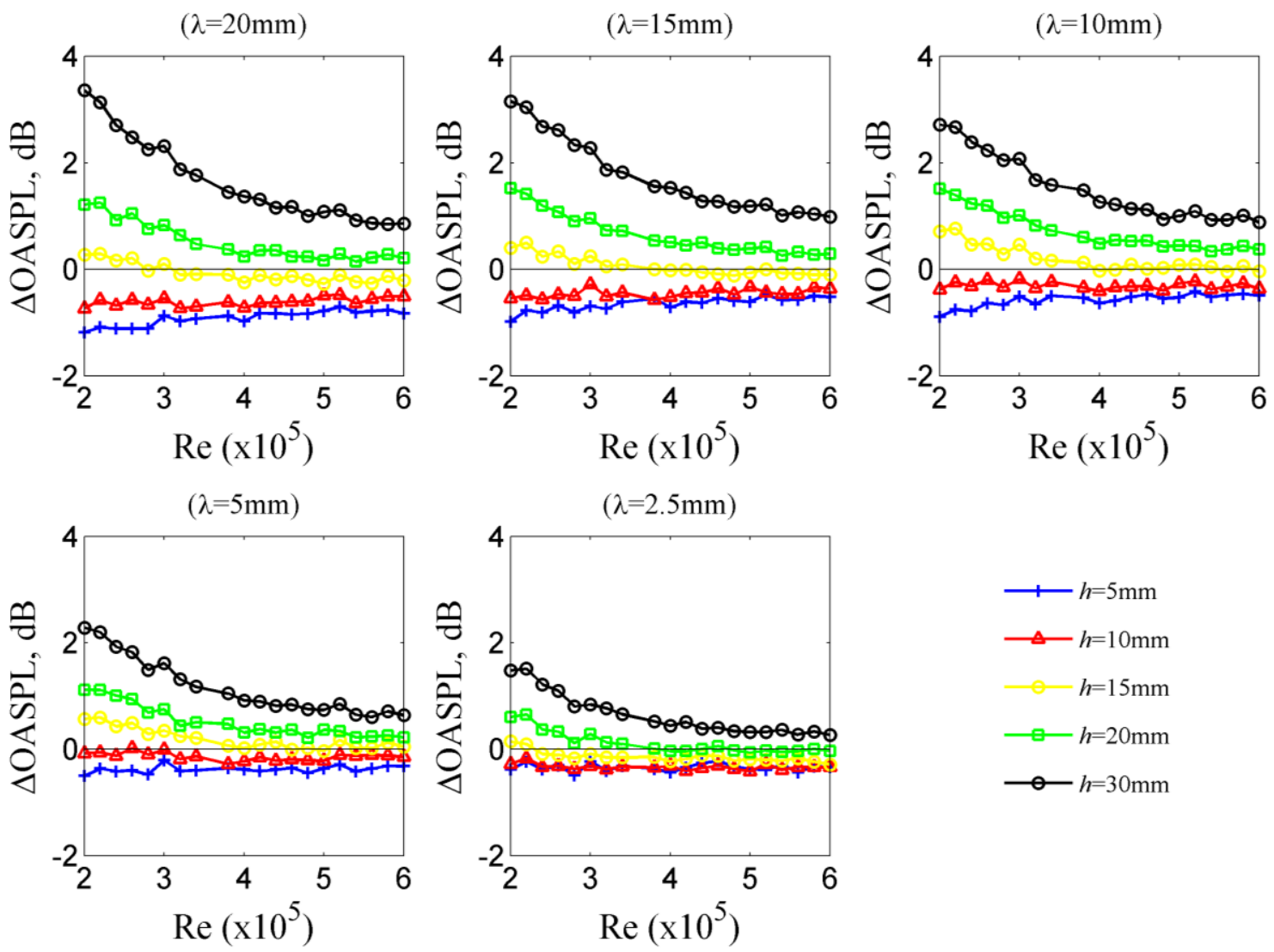

Fig. $8 \triangle O A S P L$ (dB) produced by the serrated leading edges of different $\lambda$ and $h$ at $\operatorname{Re}=2-6 \times 10^{5}\left(U_{\infty}=20-60 \mathrm{~ms}^{-1}\right)$

across $2 \times 10^{5} \leq \operatorname{Re} \leq 4 \times 10^{5}$. The reason for not investigating at higher Re is due to the consideration that fluttering might start to occur for the flat plate devices at large $\alpha$.

Figure $9 \mathrm{a}$ and $9 \mathrm{~b}$ show the SPL spectra produced by the $\lambda 0 h 0$ and $\lambda 20 h 30$, respectively, at $0^{\circ} \leq \alpha \leq 10^{\circ}$ and $\operatorname{Re}=$ $3 \times 10^{5}$. It can be seen that for the baseline $\lambda 0 h 0$, the SPL spectra are not too sensitive to the change in $\alpha$. By contrast, the SPL spectra produced by the $\lambda 20 h 30$ serrated leading edge increases with $\alpha$ up to $3 \mathrm{~dB}$ at $600 \mathrm{~Hz}<f<$ $1,600 \mathrm{~Hz}$; and up to $12 \mathrm{~dB}$ at $f \geq 1,600 \mathrm{~Hz}$. Contour maps of the $\Delta \mathrm{SPL}$ between the $\lambda 0 h 0$ and $\lambda 20 h 30$, as a function of $U_{\infty}$ and $f$, are shown in Fig. $9 \mathrm{c}$ for the different $\alpha$ case. Only minor changes in the $\Delta$ SPL are observed from $\alpha=0^{\circ}$ to $2^{\circ}$ due to the relatively small change in the 'effective' angle of attack. At $\alpha=4^{\circ}$, the level of broadband noise reduction and the corresponding frequency range start to reduce. At the same time, the increase of the high frequency noise becomes more prominent. Between $\alpha=8^{\circ}$ and $10^{\circ}$, the level of the broadband noise reduction becomes very low, whilst significant noise increase is found at the high frequency region. Overall, the serration becomes less effective as the angle of attack is increased.

\section{Some Aeroacoustic Characteristics for the Curved-Serrated Leading Edges}

Figure 10a shows some examples of the leading edge serration on owl wings. The curvature of the serration could be regarded as a unique morphology, and as stated in the Section I, another objective of this paper is to investigate whether implementing a curved-serration on the leading edge could further improve the broadband noise reduction. The hypothesis is based on the geometrical consideration that the curved-flow path within the sawtooth gap/passage will yield a longer effective serration amplitude $h^{\prime}$, which is normally longer than the $h$ counterpart for an otherwise straight-serration. As already discussed extensively in the previous section, large serration amplitude is more effective to achieve the higher $\Delta$ SPL. It is also pointed out by Kim et al. ${ }^{6}$ that the remaining contributor to the interaction noise radiation for a serrated leading edge is related to the flow dynamics at the sawtooth trough region. 


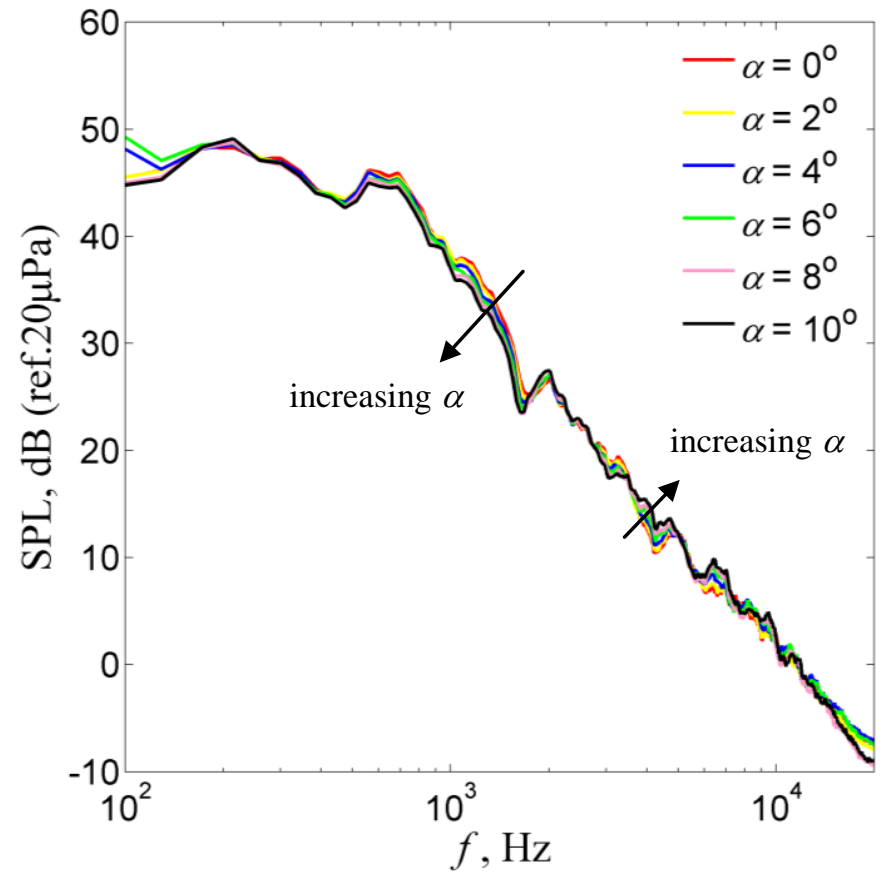

(a)

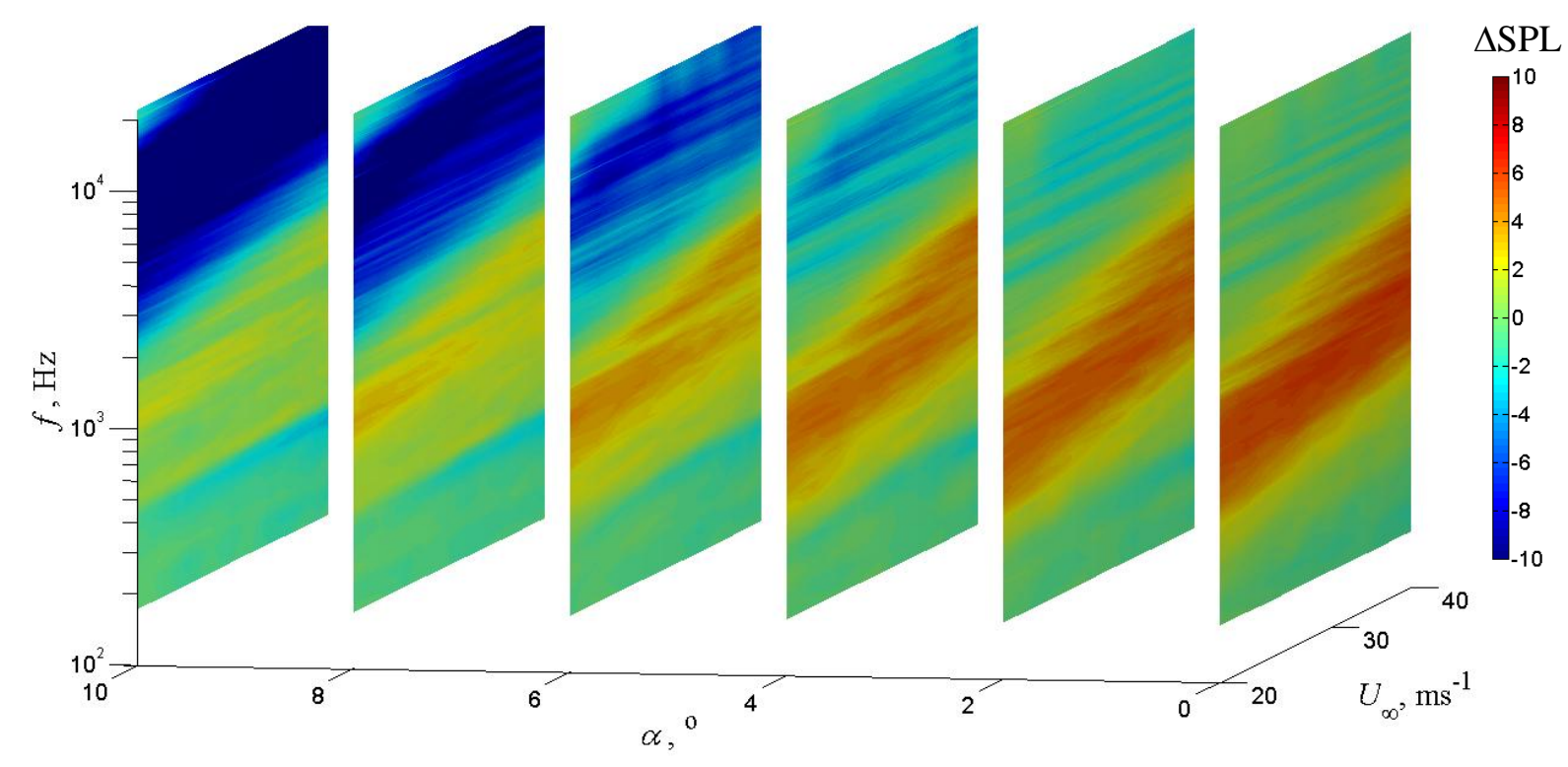

(c)

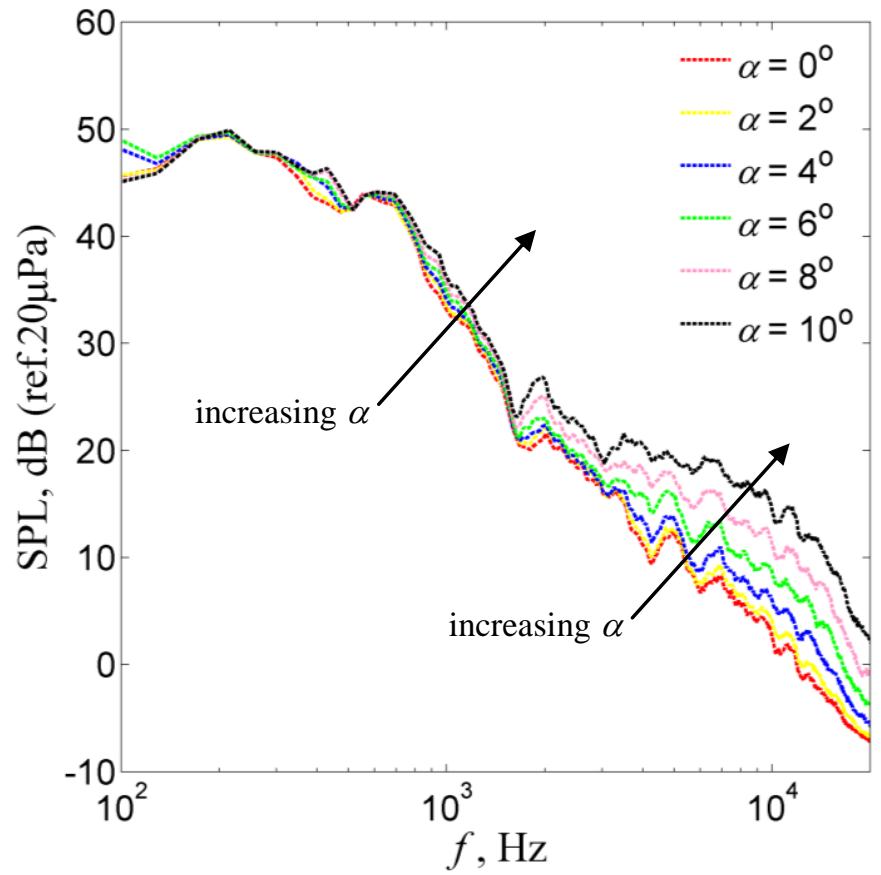

(b)

Fig. 9 SPL spectra (dB, ref $20 \mu \mathrm{Pa})$ measured at $\alpha=0^{\circ}-10^{\circ}$ and $\operatorname{Re}=3 \times 10^{5}\left(U_{\infty}=30 \mathrm{~ms}^{-1}\right)$ for (a) unmodified baseline leading edge $\lambda 0 h 0$, and (b) $\lambda 20 h 30$ serrated leading edge. (c) Sensitivity of the $\Delta \mathrm{SPL}(\mathrm{dB})$ to the $\alpha$. Contour maps are presented as a function of $U_{\infty}$ and $f$

Because each consecutive sawtooth tips of a curved leading edge serration exhibits a spanwise offset to the sawtooth trough at the downstream, the incoming turbulence structures could be shielded by the sawtooth tip. This has a potential to reduce the turbulence level at the trough, which then leads to a further reduction of the broadband interaction noise level. An example of the curved-serration implemented in the current NACA0008 aerofoil is 


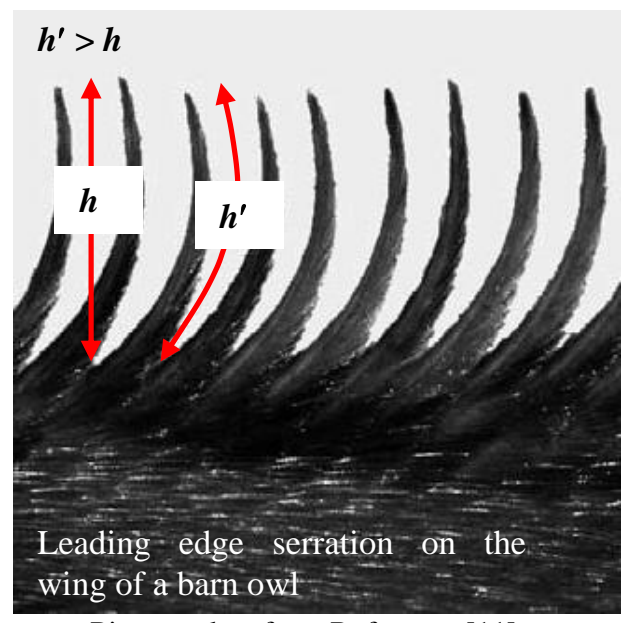

Picture taken from Reference [11].

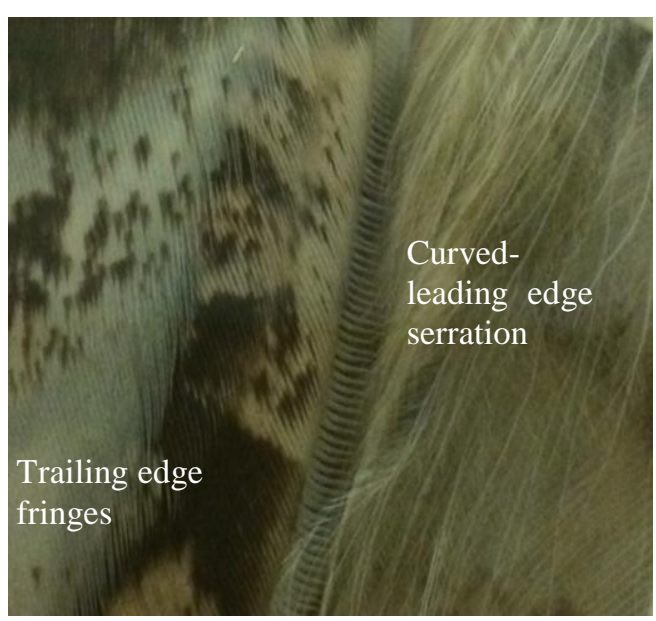

Picture taken at the Tring Natural History Museum, U.K. by the present authors

(a)

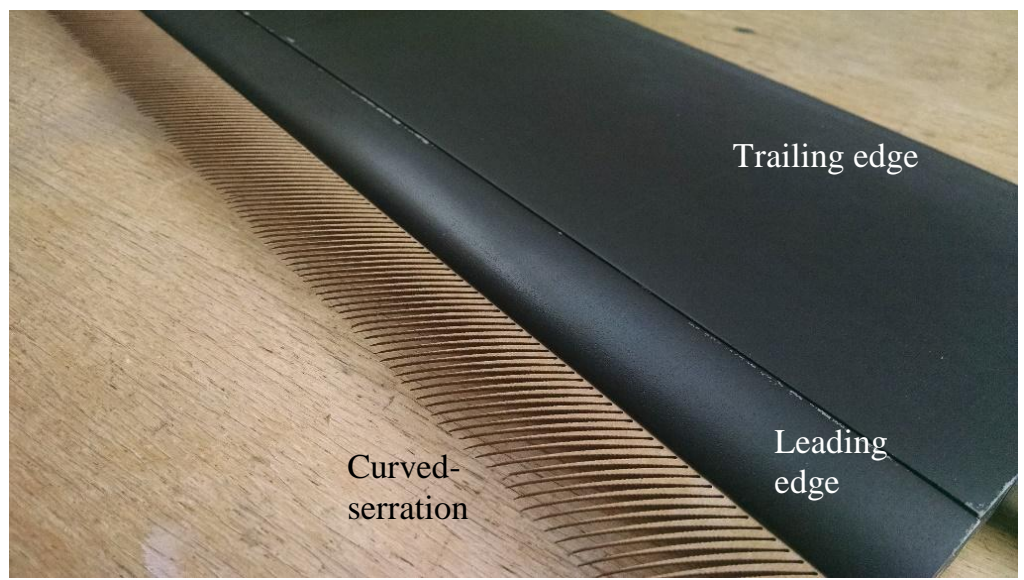

(b)

Fig. 10 (a) Examples of curved-leading edge serration on owls, and (b) curved-serrated leading edge attached to the NACA0008 model

shown in Fig. 10b. As a reminder, three additional geometric parameters could be used to describe a curvedserration. These are the inclination angle $i$, curvature $R$ and the effective serration amplitude $h^{\prime}$ (See Fig. 3b). Similarly, the experiments for the curved-serration cover $2 \times 10^{5} \leq \operatorname{Re} \leq 6 \times 10^{5}$, but it was only performed at $\alpha=0^{\circ}$.

It should be mentioned that the curve-serration only makes sense when the $h$ is sufficiently large. Therefore, this paper only includes the results of the serrated leading edges with $h=30 \mathrm{~mm}$. Figure 11a shows the SPL spectra for the $\lambda 0 h 0, \lambda 20 h 30$ (straight), $\lambda 20 h 30\left(i 15^{\circ} R 50\right), \lambda 20 h 30\left(i 15^{\circ} R 100\right), \lambda 20 h 30\left(i 30^{\circ} R 50\right)$ and $\lambda 20 h 30\left(i 30^{\circ} R 100\right)$ at $\operatorname{Re}=6 x$ $10^{5}$. A clear trend for the results in Fig. 11a is that all the curved-serration cases are incapable of matching the noise reduction performance produced by the straight-serration counterpart. For the cases when $i=30^{\circ}$, the curvedserrations even produced higher noise level than the baseline $\lambda 0 h 0$ at $f>4.8 \mathrm{kHz}$. Note that the corresponding straight-serration $\lambda 20 h 30$ (straight) produce similar SPL with the $\lambda 0 h 0$ at $f>4.8 \mathrm{kHz}$.

However, more encouraging results are obtained for the curved-serration pertaining to the $22.5 h 30$ case. As shown in Fig. $11 \mathrm{~b}$, both the $\lambda 2.5 h 30\left(i 15^{\circ} R 50\right)$ and $\lambda 2.5 h 30\left(i 15^{\circ} R 100\right)$ achieve a significant improvement of the broadband noise reduction at $1 \mathrm{kHz}<f<3.8 \mathrm{kHz}$, which is up to $5 \mathrm{~dB}$ higher than that achieved by the $22.5 h 30$ (straight) serration. In other words, they can achieve $\Delta$ SPL up to $14 \mathrm{~dB}$ when compared with the clean baseline $\lambda 0 h 0$. Moreover, the noise increase at high frequency for the $\lambda 2.5 h 30$ (straight), as observed in the previous 

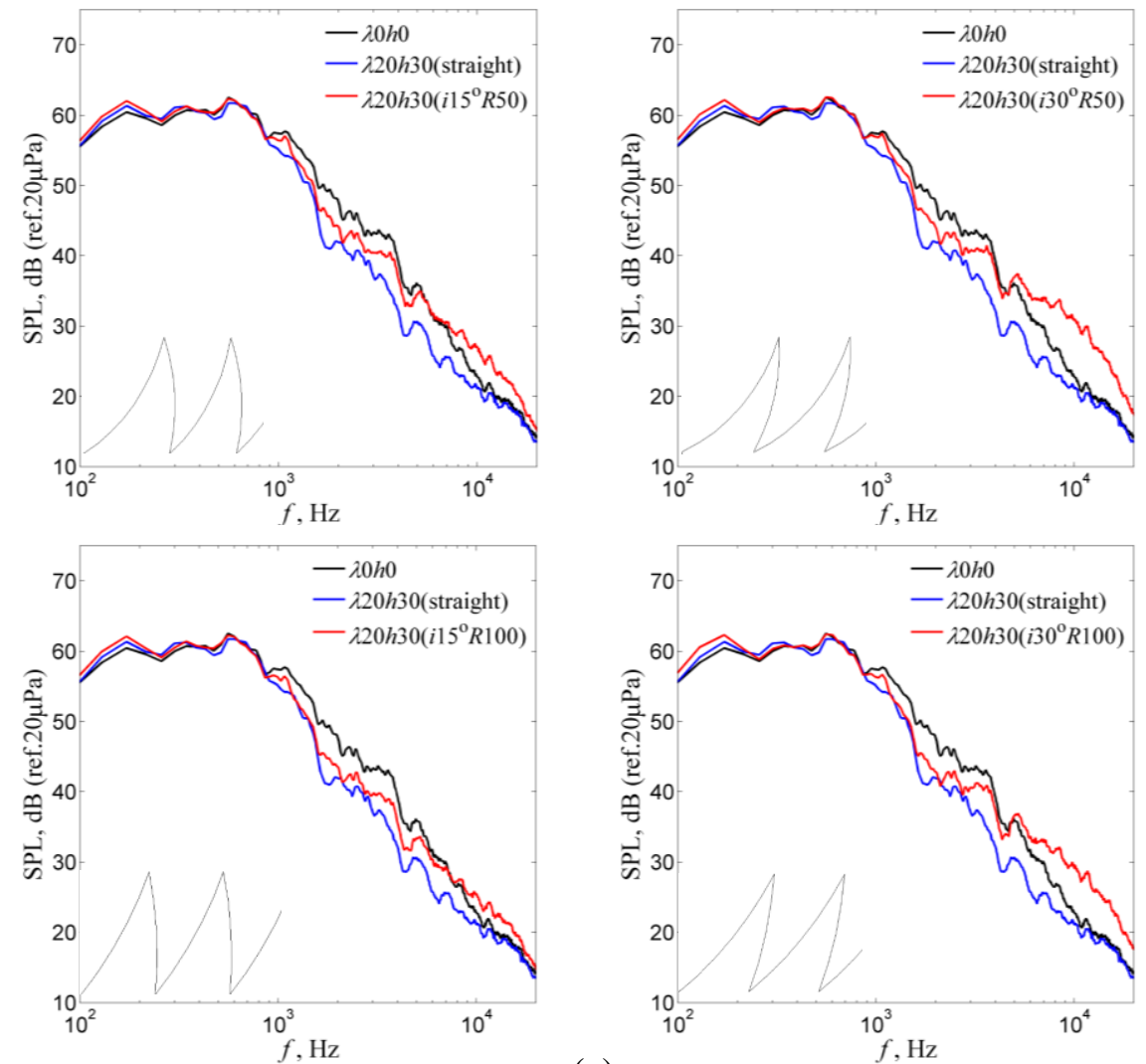

(a)
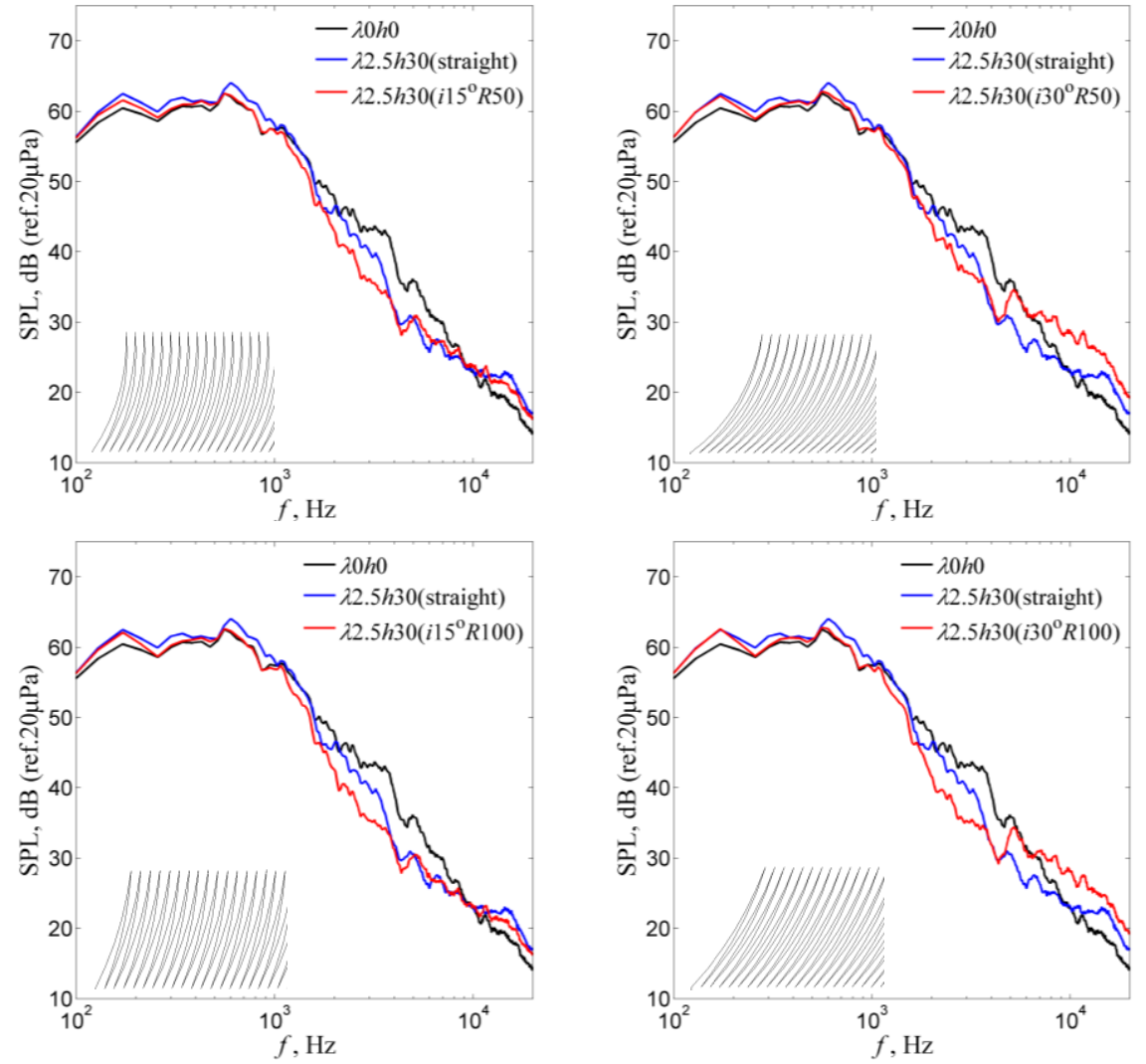

(b)

Fig. 11 Comparisons of the SPL (dB, ref $20 \mu \mathrm{Pa})$ spectra between the straight and curved serrated leading edges for the cases of (a) $\lambda 20 \mathrm{h30}$, and (b) $\lambda 2.5 \mathrm{h30}$, at $\operatorname{Re}=6 \times 10^{5}\left(U_{\infty}=60 \mathrm{~ms}^{-1}\right)$

section and is shown here again, becomes lessened at $f>19 \mathrm{kHz}$. Both the $22.5 h 30\left(i 30^{\circ} R 50\right)$ and $22.5 h 30\left(i 30^{\circ} R 100\right)$

American Institute of Aeronautics and Astronautics 

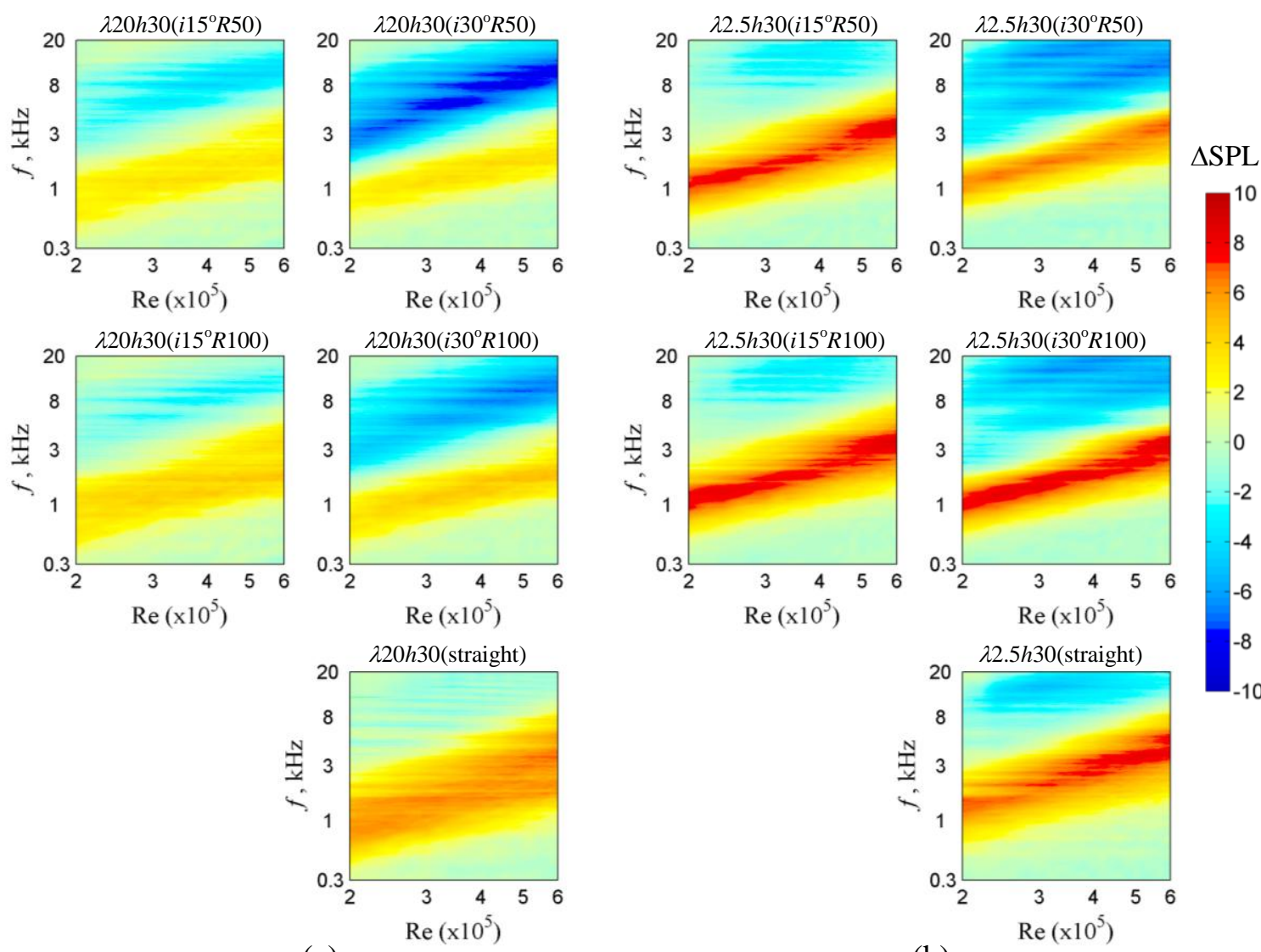

(a)

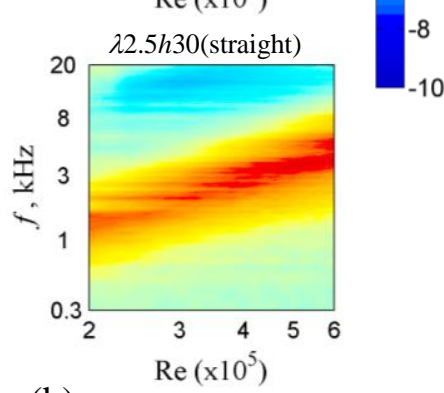

(b)

Fig. 12 Sensitivity of the $\triangle \mathrm{SPL}(\mathrm{dB})$ to the inclination angle $(i)$ and curvature radius $(R)$ of the serrated leading edge for the cases of (a) $\lambda 20 h 30$, and (b) $\lambda 2.5 h 30$, across $\operatorname{Re}=2-6 \times 10^{5}\left(U_{\infty}=20-60 \mathrm{~ms}^{-1}\right)$

can also further reduce the broadband noise to a significant level between $1 \mathrm{kHz}<f<3.8 \mathrm{kHz}$. However, this advantage is offset by the significant noise increase at $f>5 \mathrm{kHz}$.

So far, it is clear that a sufficiently large inclination angle $i\left(\right.$ e.g. $\left.i=30^{\circ}\right)$ is prone to produce significant noise increase at high frequency. The level of noise increase is even higher than those produced by the baseline $\lambda 0 h 0$ and the corresponding straight-serration. It is worth noting that at $i=30^{\circ}$, it could still outperform the straight-serration counterpart for the broadband noise in the mid-frequency region, provided that the serration wavelength $\lambda$ is small. The concept of curved-serration generally works very well for a serrated leading edge with small $\lambda$ and large $h$, as demonstrated in Fig. 11b. Another observation is that the level of broadband noise reduction is not very sensitive to the serration curvature radius $R$.

To ensure that the above observations for the curve-serration is also valid at other Re, contour maps of $\triangle \mathrm{SPL}$ as a function of Re and $f$ between (1) the baseline $\lambda 0 h 0$ and the straight-serrations; (2) the baseline $\lambda 0 h 0$ and the curvedserrations, are shown in Fig. 12. It is clear that all the above observations are also valid for other Re. To summarise from these $\Delta$ SPL contour plots, the curved-serration $\lambda 2.5 h 30\left(i 15^{\circ} R 100\right)$ outperforms the $\lambda 2.5 h 30$ (straight) in two aspects: (1) larger level of broadband noise reduction at the mid-frequency region (up to $5 \mathrm{~dB}$ higher), and (2) lower level of noise increase (about $3 \mathrm{~dB}$ less) at the high-frequency region.

Without performing the flow field investigation, there might be hints from the noise experimental data which allows us to investigate the mechanism of the further reduction in broadband noise by a suitably-optimised curvedserration. To this end, the $22.5 h \mathrm{XX}$-type serration is chosen and a correlation is studied between the maximum level of $\Delta \mathrm{SPL}_{(\max )}$ and either the $h$ (for the straight-serration case) or the $h^{\prime}$ (for the curved-serration cases) at $\mathrm{Re}=6 \times 10^{5}$. As shown in Fig. 13, the $\Delta \mathrm{SPL}_{(\max )}$ corresponding to the $\lambda 2.5 h 5$ (straight), $\lambda 2.5 h 10$ (straight), $\lambda 2.5 h 15$ (straight), $\lambda 2.5 h 20$ (straight) and $\lambda 2.5 h 25$ (straight), respectively, forms an almost linear function. For the $\lambda 2.5 h 30\left(i 15^{\circ} R 50\right)$ and 


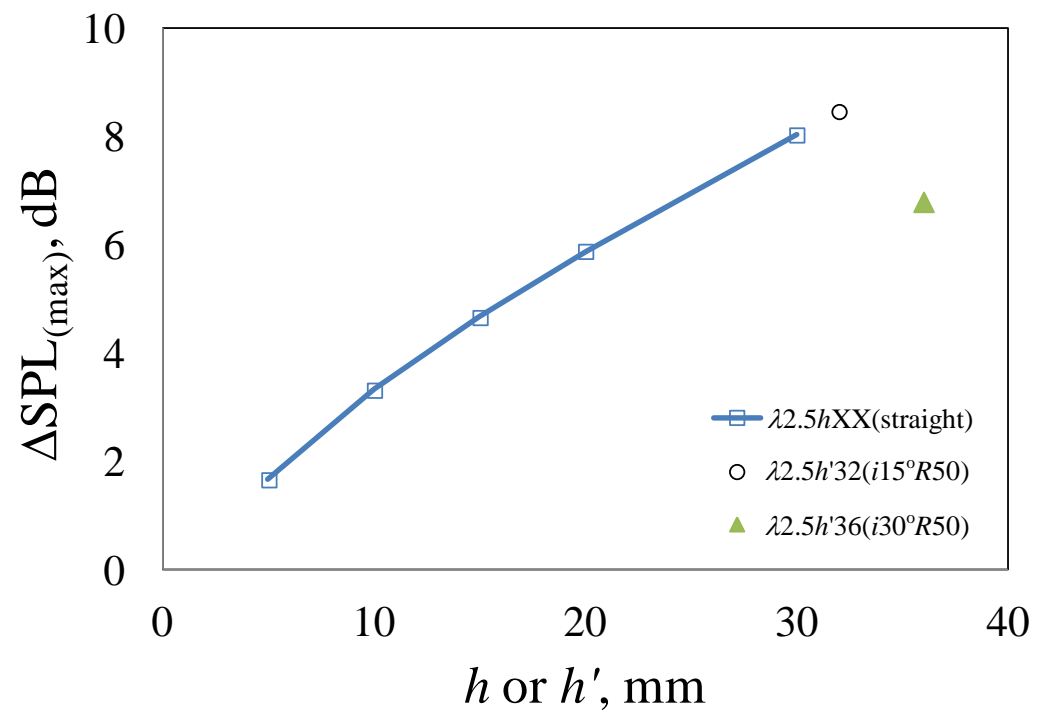

Fig. 13 Correlation of the $\triangle \mathrm{SPL}_{(\max )}$, $\mathrm{dB}$ across the serration amplitude $h$ for the straight leading edge (solid line). Distributions of the $\Delta \mathrm{SPL}_{(\max )}$ for the corresponding curved serrated leading edge against the $h^{\prime}$ are shown by the symbols $\left(i 15^{\circ} R 50\right.$ and $\left.i^{\circ} 0^{\circ} \mathrm{R5}\right)$. The case investigated here is the $\lambda 2.5 h \mathrm{XX}$ type serrated leading edge at $\operatorname{Re}=6 \times 10^{5}\left(U_{\infty}=60 \mathrm{~ms}^{-1}\right)$.

$\lambda 2.5 h 30\left(i 30^{\circ} R 50\right)$ curved-serrations, the corresponding $h^{\prime}$ are about $32 \mathrm{~mm}$ and $36 \mathrm{~mm}$, respectively. When their corresponding $\Delta \mathrm{SPL}_{(\max )}$ are inserted into Fig. 13, it was found that the $\Delta \mathrm{SPL}_{(\max )}$ for the $\lambda 2.5 h 30\left(i 15^{\circ} R 50\right)$ case would be associated with the correlation curve pertaining to the straight-serration cases. On the other hand, the $\Delta \mathrm{SPL}_{(\max )}$ for the $\lambda 2.5 h 30\left(i 30^{\circ} R 50\right)$ case cannot be described by the correlation curve.

The above results indicate that the mechanism underpinning the further broadband noise reduction by the $\lambda 2.5 h 30\left(i 15^{\circ} R 50\right)$ curved-serration is likely to be associated with the increase of the "effective" serration amplitude $h^{\prime}$ although its "normal" serration amplitude $h$ actually remains the same as the straight-serration counterpart ( $22.5 h 30)$. Despite the $22.5 h 30\left(i 30^{\circ} R 50\right)$ curved-serration actually possesses the largest $h^{\prime}$, it does not seem to achieve the expected $\Delta \mathrm{SPL}_{(\max )}$ level according to the linear function in Fig. 13. A possible explanation for this discrepancy can be referred to the oil flow visualisation results in Fig. 14. The inclination angle of the $h^{\prime}\left(i 15^{\circ} R 50\right)$ is less than the $h^{\prime}\left(i 30^{\circ} R 50\right)$. However, the oil streaks emanated from the curved-serrated leading edges, which can be regarded as the footprints resembling the upstream flow path within the sawtooth passage, demonstrate that the averaged skew angle of the oil streaks produced by the $h^{\prime}\left(i 30^{\circ} R 50\right)$ does not align well with the corresponding inclination angle of the serration. On the other hand, the averaged skew angle of the oil streaks for the $h^{\prime}\left(i 15^{\circ} R 50\right)$ is quite similar to the corresponding inclination angle of the serrated leading edge. This suggests that the real $h^{\prime}$ for the $h^{\prime}\left(i 30^{\circ} R 50\right)$ should be less than $36 \mathrm{~mm}$. Another explanation for the lack of performance of the $h^{\prime}\left(i 30^{\circ} R 50\right)$ case is that the vertical line joining the sawtooth peak to the inner surface of the adjacent curved-serration (indicated by the red lines in Fig. 14) is much shorter than the $h^{\prime}\left(i 15^{\circ} R 50\right)$ case. This indicates that the $h^{\prime}\left(i 15^{\circ} R 50\right)$ configuration will facilitate a smoother entry of the incoming flow to the sawtooth air passage, allow a better flow path in accordance to the curved passage, and prolong the interaction with the inner surface of the adjacent curved-serration. In short, the "curvature" effect will be fully maximised. A final statement about the curved-serration is that currently there is no evidence to support the hypothesis that the peak of the curved-serration can prevent the interaction between the turbulence structures and the serration troughs.

\section{Conclusion}

This paper presents the experimental results of the effect of add-on type leading edge serrations on the aeroacoustic performance of a symmetrical NACA0008 aerofoil. The focus is on the turbulence-leading edge 

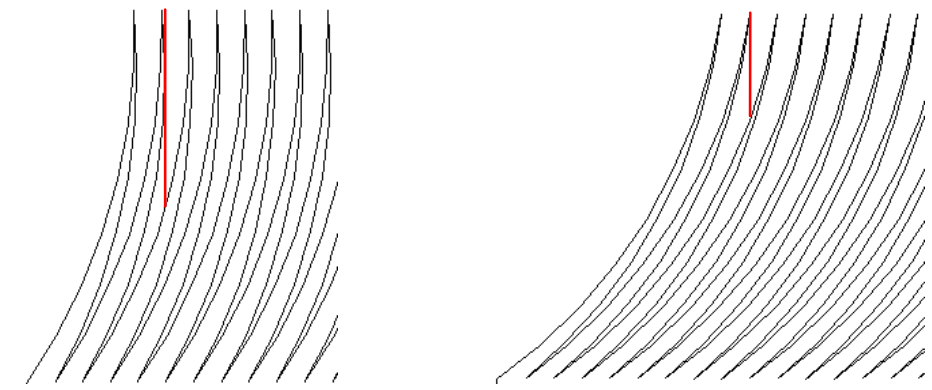

$\left.h^{\prime}{ }_{(i 15}{ }^{\mathrm{o}} \mathrm{R} 50\right)$

$<$

$\left.h^{\prime}{ }_{(i 30}^{0} R 50\right)$

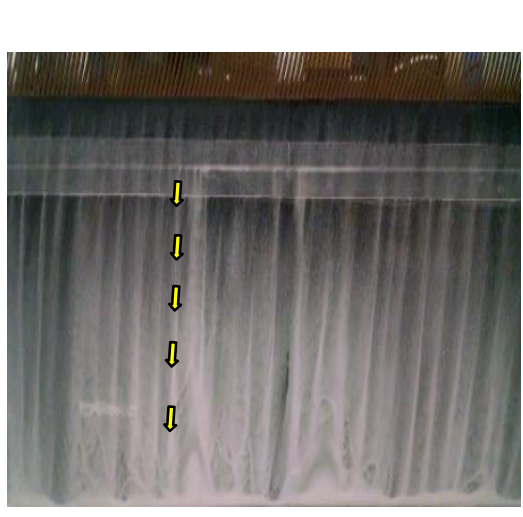

(a)

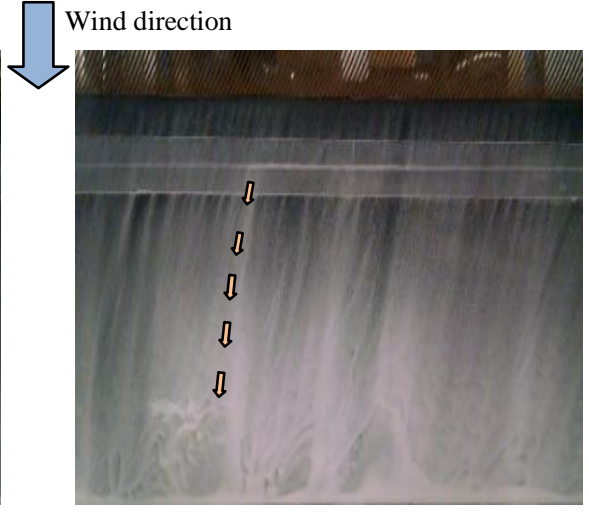

(b)

Fig. 14 Schematics and surface oil flow visualisation maps (suction side of the aerofoil) to hypothesise the flow paths within the curved serrated leading for the (a) $i 15^{\circ} R 50$, and (b) $i 30^{\circ} R 50$ cases. The serrated leading edge investigated here is the $\lambda 2.5 \mathrm{h30}$. The aerofoil was positioned at $5^{\circ}$ angle of attack (effective) at a separate wind tunnel.

interaction broadband noise. Tests have been conducted in an aeroacoustic open jet wind tunnel at Brunel University London. 25 serrated leading edges (straight) that can be adequately described by their serration wavelength $\lambda$ and serration amplitude $h$ have been investigated at Reynolds numbers between 0.2 and 0.6 millions. It was found that those with large $h$ are very effective in reducing the broadband noise levels up to about $9 \mathrm{~dB}$. However, the serration designs with smaller $h$ can produce noticeable levels of noise increase at high frequency. The effect of the $\lambda$ on the noise reduction depends on the corresponding $h$ value. For example, a particular design with both the largest $\lambda$ and $h$ has been shown to be very effective. Another one with a similar $\lambda$, but the smallest $h$ can actually degrade the performance significantly where noise increase over the baseline aerofoil becomes dominant.

The curved-serration has been shown to be able to outperform the straight-serration counterpart by a further $5 \mathrm{~dB}$ broadband noise reduction when it is optimised properly. The mechanism is due to the increase of the "effective" serration amplitude $h^{\prime}$ as a result of the curvature although its "normal" serration amplitude $h$ actually remains the same as the straight-serration counterpart. Currently, there is no evidence to support the hypothesis that the peak of the curved-serration can prevent the interaction between the grid-generated turbulence structures and the serration troughs.

Overall, the add-on type leading edge serration has been shown to be very effective in the reduction of the interaction broadband noise. The nature-inspired concept of a curved-serration at the leading edge provides an avenue for further research. 


\section{Acknowledgments}

The authors would like to thank the financial supports from the EPSRC Doctoral Training Partnership (DTP), and the EPSRC Research Grant No: EP/N018737/1 on the "Quiet Aerofoils of the Next Generation".

\section{References}

1. Staubs JK. Real airfoil effects on leading edge noise. Ph.D. thesis, Virginia Polytechnic Institute and State University, Blackburgh, VA (2008).

2. Soderman P. Aerodynamic effects of leading edge serrations on a two dimensional airfoil. NASA Technical Memorandum 1972:TM X-2643.

3. Hersh AS, Hayden RE. Aerodynamic sound radiation from lifting surfaces with and without leading-edge serrations. NASA Contract Report 1971:No. 114370.

4. Clair V, Polacsek C, Le Garrec T, Reboul G, Gruber M, Joseph.PF. Experimental and numerical investigation of turvulence-airfoil noise reduction using wavy edges. AIAA J. 2013:51: 2695-2713.

5. Narayanan S, Joseph PF, Haeri S, Kim JW, Chaitanya P, Polacsek C. Noise reduction studies from the leading edge of serrated flat plates. Proc $20^{\text {th }}$ AIAA/CEAS Aeroacoustic Conference, Atlanta, USA 2014, AIAA-2014-2320.

6. Kim JW, Haeri S, Joseph PF. On the reduction of aerofoil-turbulence interaction noise associated with wavy leading edges. J. Fluid Mech. 2016:792:526-552.

7. Biedermann TM, Chong TP, Kameier F, Paschereit CO. Statistical-empirical modeling of airfoil noise subjected to leading edge serrations. AIAA J. (in press)

8. Vathylakis A, Kim JH, Chong TP. Design of a low-noise aeroacosutic wind tunnel facility at Brunel University. Proc $20^{\text {th }}$ AIAA/CEAS Aeroacoustic Conference, Atlanta, USA 2014 AIAA-2014-3288.

9. Chong TP, Vathylakis A, McEwen A, Kemsley F, Muhammad C, Siddiqi S. Aeroacoustic and aerodynamic performances of an aerofoil subjected to sinusoidal leading edges. Proc $21^{\text {st }}$ AIAA/CEAS Aeroacoustic Conference, Dallas, USA 2015 AIAA-2015-2200.

10. Laws EM, Livesey JL. Flow through screens, Annu. Rev. Fluid Mech. 1978: 10: 247-266.

11. Bachmann T, Klan S, Baumgartner W, Klaas M, Schroder W, Wagner H. Morphometric characterisation of wing feathers of the barn owl Tyto alba pratincola and the pigeon Columba livia. Frontiers in Zoology 2007:4:23 